\title{
GLOBAL REGULAR SOLUTIONS TO THE NAVIER-STOKES EQUATIONS IN A CYLINDER
}

\author{
WOJCIECH M. ZAJĄCZKOWSKI \\ Institute of Mathematics, Polish Academy of Sciences \\ Śniadeckich 8, 00-956 Warszawa, Poland \\ E-mail: wz@impan.gov.pl \\ and \\ Institute of Mathematics and Cryptology, Cybernetics Faculty \\ Military University of Technology \\ S. Kaliskiego 2, 00-908 Warszawa, Poland
}

\begin{abstract}
The existence and uniqueness of solutions to the Navier-Stokes equations in a cylinder $\Omega$ and with boundary slip conditions is proved. Assuming that the azimuthal derivative of cylindrical coordinates and azimuthal coordinate of the initial velocity and the external force are sufficiently small we prove long time existence of regular solutions such that the velocity belongs to $W_{5 / 2}^{2,1}(\Omega \times(0, T))$ and the gradient of the pressure to $L_{5 / 2}(\Omega \times(0, T))$. We prove the existence of solutions without any restrictions on the lengths of the initial velocity and the external force.
\end{abstract}

1. Introduction. We examine the following problem (see [7]):

$$
\begin{array}{cl}
v_{, t}+v \cdot \nabla v-\operatorname{div} \mathbb{T}(v, p)=f & \text { in } \Omega^{T}=\Omega \times(0, T), \\
\operatorname{div} v=0 & \text { in } \Omega^{T}, \\
v \cdot \bar{n}=0 & \text { on } S^{T}=S \times(0, T), \\
\bar{n} \cdot \mathbb{D}(v) \cdot \bar{\tau}_{\alpha}=0, \quad \alpha=1,2, & \text { on } S^{T}, \\
\left.v\right|_{t=0}=v(0) & \text { in } \Omega,
\end{array}
$$

where $\Omega \subset \mathbb{R}^{3}$ is a cylinder with the boundary $S$.

By $v=\left(v_{1}, v_{2}, v_{3}\right) \in \mathbb{R}^{3}$ we denote the velocity of the fluid, $p \in \mathbb{R}$ the pressure, $f=\left(f_{1}, f_{2}, f_{3}\right) \in \mathbb{R}^{3}$ the external force, $\bar{n}$ is the unit outward vector normal to $S, \bar{\tau}_{\alpha}$,

2000 Mathematics Subject Classification: 35Q35, 35K20, 76D05, 76D03.

Key words and phrases: global existence of regular solutions, Navier-Stokes equations, incompressible fluid, solutions with large initial velocity and external force, solutions close to axially symmetric.

The paper is in final form and no version of it will be published elsewhere. 
$\alpha=1,2$, are tangent to $S$. Moreover, the dot $\cdot$ denotes the scalar product in $\mathbb{R}^{3}$. By $\mathbb{T}(v, p)$ we denote the stress tensor of the form

$$
\mathbb{T}(v, p)=\nu \mathbb{D}(v)-p I,
$$

where $\nu$ is the constant viscosity coefficient, $\mathbb{D}(v)$ the dilatation tensor of the form

$$
\mathbb{D}(v)=\left\{v_{i, x_{j}}+v_{j, x_{i}}\right\}_{i, j=1,2,3},
$$

and $I$ is the unit matrix.

Let $\left(x_{1}, x_{2}, x_{3}\right)$ be a local Cartesian system such that the $x_{3}$ axis is the axis of the cylinder $\Omega$. Let $(r, \varphi, z)$ be the cylindrical coordinates such that $x_{1}=r \cos \varphi, x_{2}=r \sin \varphi$, $x_{3}=z$.

Let for given $R>0, a>0$

$$
\Omega=\left\{x \in \mathbb{R}^{3}: r<R, \varphi \in[0,2 \pi], z \in(-a, a)\right\},
$$

$S=S_{1} \cup S_{2}$ where

$$
\begin{gathered}
S_{1}=\left\{x \in \mathbb{R}^{3}: r=R, \varphi \in[0,2 \pi], z \in(-a, a)\right\}, \\
S_{2}=\left\{x \in \mathbb{R}^{3}: r<R, \varphi \in[0,2 \pi], z \text { is either }-a \text { or } a\right\} .
\end{gathered}
$$

To prove the existence of solutions to problem (1.1) we apply the Leray-Schauder fixed point theorem. For this purpose we define a mapping $\Phi$ whose fixed point is a solution to problem (1.1).

Let

$$
\begin{gathered}
\mathfrak{M}\left(\Omega^{T}\right)=L_{4}\left(0, T ; L_{\infty}(\Omega)\right) \cap L_{2}\left(0, T ; W_{3}^{1}(\Omega)\right) \cap L_{\infty}\left(0, T ; W_{2,-\delta}^{1}(\Omega)\right) \\
\cap L_{\infty}\left(0, T ; L_{4,-\delta}(\Omega)\right) \cap L_{2}\left(0, T ; W_{6 /\left(1-2 \varepsilon_{0}\right)}^{1}(\Omega)\right),
\end{gathered}
$$

where $\delta \in(0,1 / 2), \varepsilon_{0} \in(0,1 / 2)$.

Now we construct the mapping $\Phi$. Let $v^{\prime} \in \mathfrak{M}\left(\Omega^{T}\right)$ be given. By Lemmas 3.1-3.4 and inequalities (4.4), (4.13) there exists a solution to problem below (2.12) denoted by $\tilde{v}=\tilde{v}\left(v^{\prime}\right) \in \mathfrak{M}_{0}\left(\Omega^{T}\right)=L_{10}\left(\Omega^{T}\right) \cap L_{10 / 3}\left(0, T ; W_{10 / 3}^{1}(\Omega)\right)$. The relation $\tilde{v}=\tilde{v}\left(v^{\prime}\right)$ defines the mapping

$$
\Phi_{1}: \mathfrak{M}\left(\Omega^{T}\right) \ni v^{\prime} \mapsto \Phi_{1}\left(v^{\prime}\right)=\tilde{v} \in \mathfrak{M}_{0}\left(\Omega^{T}\right) .
$$

Then problem (5.1) generates the mapping $\Phi_{2}$,

$$
v=\Phi_{2}(\tilde{v}, \lambda)
$$

such that

$$
\Phi_{2}: \mathfrak{M}_{0}\left(\Omega^{T}\right) \times[0,1] \rightarrow \mathfrak{M}_{*}\left(\Omega^{T}\right)=W_{5 / 2}^{2,1}\left(\Omega^{T}\right) \subset \mathfrak{M}\left(\Omega^{T}\right) .
$$

From (1.4) and (1.5) we define the transformation

$$
\Phi: \mathfrak{M}\left(\Omega^{T}\right) \times[0,1] \rightarrow \mathfrak{M}_{*}\left(\Omega^{T}\right)
$$

by

$$
\Phi\left(v^{\prime}, \lambda\right)=\Phi_{2}\left(\Phi_{1}\left(v^{\prime}\right), \lambda\right) .
$$

From Lemmas 5.1 and 5.2 we have that $\Phi$ is uniformly continuous and compact. Moreover, index $\left.\Phi\right|_{\lambda=0}=1$. 
Let us introduce the vectors: $\bar{e}_{r}=(\cos \varphi, \sin \varphi, 0), \bar{e}_{\varphi}=(-\sin \varphi, \cos \varphi, 0), \bar{e}_{z}=$ $(0,0,1)$ connected with the cylindrical coordinates $r, \varphi, z$, respectively. The cylindrical coordinates of any vector $u$ are $u_{r}=u \cdot \bar{e}_{r}, u_{\varphi}=u \cdot \bar{e}_{\varphi}, u_{z}=u \cdot \bar{e}_{z}$. Let $g=$ $f_{r, \varphi} \bar{e}_{r}+f_{\varphi, \varphi} \bar{e}_{\varphi}+f_{z, \varphi} \bar{e}_{z}, h=v_{r, \varphi} \bar{e}_{r}+v_{\varphi, \varphi} \bar{e}_{\varphi}+v_{z, \varphi} \bar{e}_{z}, F=\operatorname{rot} f, F^{\prime}=\left(F_{r}, F_{z}\right), \alpha=\operatorname{rot} v$, $\alpha^{\prime}=\left(\alpha_{r}, \alpha_{z}\right), w=v_{\varphi}$. Assume that the following quantities are finite:

$$
\begin{aligned}
X(t)= & \|g\|_{L_{2,-\left(1+\varepsilon_{*}\right)}\left(\Omega^{t}\right)}+\left\|f_{\varphi}\right\|_{L_{2,-\left(1 / 2+\varepsilon_{0}\right)}\left(\Omega^{t}\right)}+\left\|F^{\prime}\right\|_{L_{2}\left(0, t ; L_{6 / 5}(\Omega)\right)} \\
& +\|h(0)\|_{\left.H_{-\left(1+\varepsilon_{*}\right.}^{1}\right)}(\Omega)+\|w(0)\|_{H_{0}^{1}(\Omega)}+\left\|\alpha^{\prime}(0)\right\|_{L_{2}(\Omega)}+\left\|v_{, z}(0)\right\|_{L_{2}(\Omega)}<\infty, \\
Y_{1}(t)= & \left\|F_{\varphi}\right\|_{L_{2,-1}\left(\Omega^{t}\right)}+\left\|\alpha_{\varphi}(0)\right\|_{L_{2,-1}\left(\Omega^{t}\right)}<\infty \\
Y_{2}(t)= & \|f\|_{L_{5 / 2}\left(\Omega^{t}\right)}+\|v(0)\|_{W_{5 / 2}^{6 / 5}(\Omega)}<\infty
\end{aligned}
$$

where $\varepsilon_{*}>0$.

Let $\varphi_{1}$ be a nonnegative positive function which appears in (4.1). Let $A=\sigma\left[\varphi_{1}(0) Y_{1}^{2}+\right.$ $c_{1} Y_{2}$ ], where $\sigma \geq 2$ and $c_{1}$ is the constant from (4.1). Then for sufficiently small $X$ Lemma 4.2 implies the estimate for a fixed point of mapping $\Phi$,

$$
\|v\|_{W_{5 / 2}^{2,1}\left(\Omega^{T}\right)}+\|\nabla p\|_{L_{5 / 2}\left(\Omega^{T}\right)} \leq A .
$$

Hence, the Leray-Schauder fixed point theorem implies

THEOREM 1.1. Let (1.7) hold. Let $X$ be sufficiently small. Then there exists a solution to problem (1.1) such that $v \in W_{5 / 2}^{2,1}\left(\Omega^{T}\right), \nabla p \in L_{5 / 2}\left(\Omega^{T}\right)$ and (1.8) holds.

2. Notation and auxiliary results. By $c$ we denote generic constants and by $c(\sigma)$ we denote generic positive increasing functions depending on $\sigma$.

To simplify considerations we introduce

$$
\begin{gathered}
|u|_{p, Q}=\|u\|_{L_{p}(Q)}, \quad Q \in\left\{\Omega, S, \Omega^{T}, S^{T}\right\}, \quad p \in[1, \infty], \\
\|u\|_{s, Q}=\|u\|_{H^{s}(Q)}, \quad Q \in\{\Omega, S\}, \quad s \in \mathbb{R}_{+} \cup\{0\}, \\
\|u\|_{s, Q}=\|u\|_{W_{2}^{s, s / 2}(Q)}, \quad Q \in\left\{\Omega^{T}, S^{T}\right\}, \quad s \in \mathbb{R}_{+} \cup\{0\},
\end{gathered}
$$

where $\|u\|_{0, Q}=|u|_{2, Q}$.

Moreover, $|u|_{p, q, Q^{T}}=\|u\|_{L_{q}\left(0, T ; L_{p}(Q)\right)}$ and

$$
|u|_{p, q, \mu, Q^{T}}=\|u\|_{L_{q}\left(0, T ; L_{p, \mu}(Q)\right)}, \quad Q \in\{\Omega, S\}, \quad p, q \in[1, \infty], \quad \mu \in \mathbb{R} .
$$

Let us introduce

$$
\|v\|_{V_{2}^{s}\left(\Omega^{T}\right)}=\sup _{t \leq T}\|v(t)\|_{H^{s}(\Omega)}+\left(\int_{0}^{T}\|\nabla v(t)\|_{H^{s}(\Omega)}^{2} d t\right)^{1 / 2}
$$

Finally, we introduce weighted spaces. Let $L_{p, \mu}(Q)$ be the set of functions $u$ such that

$$
\|u\|_{L_{p, \mu}(Q)}=\left(\int_{Q}|u|^{p} r^{p \mu} d x\right)^{1 / p}<\infty, \quad p \in[1, \infty], \quad \mu \in \mathbb{R}, \quad Q \in\left\{\Omega, S, \Omega^{T}, S^{T}\right\}
$$

with the notation

$$
|u|_{p, \mu, Q}=\|u\|_{L_{p, \mu}(Q)} .
$$


Let us define $H_{\mu}^{s}(Q)$ for $Q \in\{\Omega, S\}, s \in \mathbb{Z}_{+} \cup\{0\}, \mu \in \mathbb{R}$ by

$$
\|u\|_{H_{\mu}^{s}(Q)}=\left(\sum_{|\alpha| \leq s} \int_{Q}\left|D_{x}^{\alpha} u\right|^{2} r^{2(\mu-s+|\alpha|)} d Q\right)^{1 / 2}<\infty
$$

and $H_{\mu}^{s, s / 2}(Q)$ for $Q \in\left\{\Omega^{T}, S^{T}\right\}, \frac{s}{2} \in \mathbb{Z}_{+} \cup\{0\}, \mu \in \mathbb{R}$ by

$$
\|u\|_{H_{\mu}^{s, s / 2}(Q)}=\left(\sum_{|\alpha|+2 i \leq s} \int_{Q}\left|D_{x}^{\alpha} \partial_{t}^{i} u\right|^{2} r^{2(\mu-s+|\alpha|+2 i)} d Q\right)^{1 / 2}<\infty .
$$

To simplify notation we introduce

$$
\|u\|_{s, \mu, Q}=\|u\|_{H_{\mu}^{s}(Q)} \quad \text { for } \quad Q \in\{\Omega, S\}
$$

and

$$
\|u\|_{s, \mu, Q}=\|u\|_{H_{\mu}^{s, s / 2}(Q)} \text { for } Q \in\left\{\Omega^{T}, S^{T}\right\} .
$$

Finally, we define

$$
V_{p, \mu}^{s}(\Omega)=\left\{u:\|u\|_{V_{p, \mu}^{s}(\Omega)}=\left(\sum_{|\alpha| \leq s} \int_{\Omega}\left|D_{x}^{\alpha} u\right|^{p} r^{p(\mu-s+|\alpha|)} d x\right)^{1 / p}<\infty\right\} .
$$

Now we recall inequalities and imbedding theorems used in this paper.

From [5] we have the imbedding

$$
\|u\|_{V_{q, \beta+s-l+\frac{n}{p}-\frac{n}{q}}^{s}(\Omega)} \leq c\|u\|_{V_{p, \beta}^{l}(\Omega)}, \quad \Omega \subset \mathbb{R}^{n},
$$

and $s-l+\frac{n}{p}-\frac{n}{q} \leq 0$.

From Lemmas 4.2.3 and 4.2.4 in [7] we have the following Korn inequality:

$$
\|v\|_{1, \Omega}^{2} \leq c\left(E_{\Omega}(v)+\left|\int_{\Omega} v_{\varphi}(0) r d x\right|^{2}+\left|\int_{\Omega^{t}} f_{\varphi} r d x d t^{\prime}\right|^{2}\right)
$$

where

$$
E_{\Omega}(v)=\int_{\Omega}\left(v_{i, x_{j}}+v_{j, x_{i}}\right)^{2} d x
$$

and the summation convention over the repeated indices is assumed. Moreover, to show (2.2) we used the conservation law

$$
\int_{\Omega} v_{\varphi}(t) r d x=\int_{\Omega^{t}} f_{\varphi} r d x d t^{\prime}+\int_{\Omega} v_{\varphi}(0) r d x
$$

In view of (2.2) we have the following energy inequality for solutions to problem (1.1) Lemma 2.1. Assume that $v(0) \in L_{2}(\Omega), v_{\varphi} \in L_{2}\left(\Omega^{t}\right), f \in L_{2}\left(\Omega^{t}\right) \cap L_{2,1}\left(\Omega^{t}\right), t \leq T$. Then

$$
\|v\|_{V_{2}^{0}\left(\Omega^{t}\right)} \leq c\left(|v(0)|_{2, \Omega}+|f|_{2, \Omega^{t}}+\left|v_{\varphi}\right|_{2, \Omega^{t}}\right), \quad t \leq T
$$

where $c$ does not depend on $T$.

Let us introduce the quantities

$$
\begin{gathered}
h=v_{r, \varphi} \bar{e}_{r}+v_{\varphi, \varphi} \bar{e}_{\varphi}+v_{z, \varphi} \bar{e}_{z}, \quad q=p_{, \varphi} \\
\alpha=\operatorname{rot} v, \quad \chi=\alpha_{\varphi}, \quad w=v_{\varphi}, \quad F=\operatorname{rot} f \\
u=v_{\varphi, z}, \quad g=f_{r, \varphi} \bar{e}_{r}+f_{\varphi, \varphi} \bar{e}_{\varphi}+f_{z, \varphi} \bar{e}_{z} .
\end{gathered}
$$


From Section 4.3 in [7] we have the following problem for $\chi$,

$$
\begin{aligned}
& \chi_{, t}+v \cdot \nabla \chi+\left(v_{r, r}+v_{z, z}\right) \chi-\nu\left[\left(r\left(\frac{\chi}{r}\right)_{, r}\right)_{, r}+\frac{1}{r^{2}} \chi_{, \varphi \varphi}+\chi_{, z z}+2\left(\frac{\chi}{r}\right)_{, r}\right] \\
& =\frac{2 \nu}{r}\left(-h_{\varphi, z}+\frac{1}{r} h_{z, \varphi}\right)-\frac{1}{r}\left(w_{, z} h_{r}-w_{, r} h_{z}+\frac{w}{r} h_{z}\right)+\frac{2}{r} v_{\varphi} v_{\varphi, z}+F_{\varphi}, \\
& \left.\chi\right|_{S}=0 \\
& \left.\chi\right|_{t=0}=\chi(0) \text {, }
\end{aligned}
$$

where $v, h, w$ are treated as given functions.

Moreover, from Section 4.3 in [7] we obtain the problem for $w$,

$$
\begin{array}{cc}
w_{, t}+v \cdot \nabla w+\frac{v_{r}}{r} w-\nu \Delta w+\nu \frac{w}{r^{2}}=\frac{1}{r} q+\frac{2 \nu}{r^{2}} h_{r}+f_{\varphi} & \text { in } \Omega^{T}, \\
w_{, r}=\frac{1}{R} w & \text { on } S_{1}^{T}, \\
w_{, z}=0 & \text { on } S_{2}^{T}, \\
\left.w\right|_{t=0}=w(0) & \text { in } \Omega,
\end{array}
$$

where $v, q, h$ are treated as given functions.

We need also the relations

$$
\begin{aligned}
\alpha_{r} & =\frac{h_{z}}{r}-w_{, z} \\
\alpha_{\varphi} & =v_{r, z}-v_{z, r} \equiv \chi, \\
\alpha_{z} & =w_{, r}+\frac{1}{r} w-\frac{1}{r} h_{r} .
\end{aligned}
$$

Finally we need the following problems for $\alpha_{r}$ (see (5.2.3) in [7])

$$
\begin{array}{cc}
\alpha_{r, t}+v \cdot \nabla \alpha_{r}-\left(v_{r, r} \alpha_{r}+v_{r, z} \alpha_{z}\right)-\frac{\chi}{r} h_{r}-\nu \Delta \alpha_{r} & \\
+\nu \frac{\alpha_{r}}{r^{2}}=-\frac{2 \nu}{r^{2}}\left(h_{r, z}-h_{z, r}\right)+F_{r} & \text { in } \Omega^{T}, \\
\alpha_{r, r}=-\frac{1}{R^{2}} h_{z}-\frac{1}{R} w_{, z} & \text { on } S_{1}^{T}, \\
\alpha_{r}=0 & \text { on } S_{2}^{T}, \\
\left.\alpha_{r}\right|_{t=0}=\alpha_{r}(0) & \text { in } \Omega
\end{array}
$$

and for $\alpha_{z}($ see $(5.2 .5)$ in [7])

$$
\begin{array}{cc}
\alpha_{z, t}+v \cdot \nabla \alpha_{z}-\left(v_{z, r} \alpha_{r}+v_{z, z} \alpha_{2}\right)-\frac{\chi}{r} h_{z} & \\
-\nu \Delta \alpha_{z}=F_{2} & \text { in } \Omega^{T}, \\
\alpha_{z}=\frac{2}{R} w & \text { on } S_{1}^{T}, \\
\alpha_{z, z}=0 & \text { on } S_{2}^{T}, \\
\left.\alpha_{z}\right|_{t=0}=\alpha_{z}(0) & \text { in } \Omega .
\end{array}
$$

Differentiating (2.6) with respect to $z$ yields the problem 


$$
\begin{array}{rlrl}
u_{, t}+v \cdot \nabla u+\frac{v_{r}}{r} u-\nu \Delta u & +\nu \frac{u}{r^{2}}=-v_{, z} \cdot \nabla w-\frac{v_{r, z}}{r} w & \\
+f_{\varphi, z}+\frac{1}{r} q_{, z}+\frac{2 \nu}{r^{2}} h_{r, z} & & \text { in } \Omega^{T}, \\
u_{, r}=\frac{1}{R} u & \text { on } S_{1}^{T}, \\
u=0 & \text { on } S_{2}^{T}, \\
\left.u\right|_{t=0}=u(0) & \text { in } \Omega,
\end{array}
$$

where the functions $v, w, q$ and $h$ are treated as given.

Next, for a given $v$ we see that $(h, q)$ is a solution to the problem

$$
\begin{array}{cc}
h_{, t}-\operatorname{div} \mathbb{T}(h, q)=-v \cdot \nabla h-h \cdot \nabla v+g \equiv G & \text { in } \Omega^{T}, \\
\operatorname{div} h=0 & \text { in } \Omega^{T}, \\
\bar{n} \cdot h=0, \quad \bar{n} \cdot \mathbb{T}(h, q) \cdot \bar{\tau}_{\alpha}=0, \quad \alpha=1,2 & \text { on } S^{T}, \\
\left.h\right|_{t=0}=h(0) & \text { in } \Omega .
\end{array}
$$

Finally, we consider the elliptic problem

$$
\begin{array}{ll}
\operatorname{rot} v=\alpha & \text { in } \Omega, \\
\operatorname{div} v=0 & \text { in } \Omega, \\
v \cdot \bar{n}=0 & \text { on } S .
\end{array}
$$

Lemma 2.2. Assume that $g \in L_{2}\left(0, T ; L_{6 / 5}(\Omega)\right), h(0) \in L_{2}(\Omega), v \in L_{2}\left(0, T ; W_{3}^{1}(\Omega)\right)$. Then solutions to problem (2.11) satisfy

$$
\begin{gathered}
|h(t)|_{2, \Omega}+\nu_{*}\|h\|_{L_{2}\left(0, t ; H^{1}(\Omega)\right)} \leq c \exp \left(\left|v_{, x}\right|_{3,2, \Omega^{t}}^{2}\right)\left[\mathbf{l} g \mathbf{|}_{6 / 5,2, \Omega^{t}}+|h(0)|_{2, \Omega}\right], \\
t \leq T,
\end{gathered}
$$

where $c$ does not depend on $T$ and $0<\nu_{*}<\nu$.

Proof. Multiplying $(2.11)_{1}$ by $h$, integrating over $\Omega$, and using the boundary conditions yields

$$
\frac{1}{2} \frac{d}{d t}|h|_{2, \Omega}^{2}+\nu|\mathbb{D}(h)|_{2, \Omega}^{2} \leq \int_{\Omega}|h \cdot \nabla v \cdot h| d x+\int_{\Omega}|g \cdot h| d x .
$$

Using the Korn inequality $\|h\|_{1, \Omega} \leq c|\mathbb{D}(h)|_{2, \Omega}$, and applying the Hölder and Young inequalities we have

$$
\frac{1}{2} \frac{d}{d t}|h|_{2, \Omega}^{2}+\nu_{*}\|h\|_{1, \Omega}^{2} \leq \varepsilon_{1}|h|_{6, \Omega}^{2}+c\left(1 / \varepsilon_{1}\right)|\nabla v|_{3, \Omega}^{2}|h|_{2, \Omega}^{2}+\varepsilon_{2}|h|_{6, \Omega}^{2}+c\left(1 / \varepsilon_{2}\right)|g|_{6 / 5, \Omega}^{2},
$$

where $\nu_{*}<\nu$.

For sufficiently small $\varepsilon_{1}$ and $\varepsilon_{2}$ we obtain

$$
\frac{d}{d t}|h|_{2, \Omega}^{2}+\nu_{*}\|h\|_{1, \Omega}^{2} \leq c|\nabla v|_{3, \Omega}^{2}|h|_{2, \Omega}^{2}+c|g|_{6 / 5, \Omega}^{2}
$$

Integrating the above with respect to $t$ implies (2.13). This ends the proof.

Let $\delta \in(0,1)$ and

$$
\mathfrak{N}\left(\Omega^{T}\right)=L_{\infty}\left(0, T ; L_{4,-\delta}(\Omega)\right) \cap L_{\infty}\left(0, T ; W_{2,-\delta}^{1}(\Omega)\right) \cap L_{2}\left(0, T ; W_{3}^{1}(\Omega)\right) .
$$


LEMMA 2.3. Let $v \in \mathfrak{N}\left(\Omega^{T}\right), \delta \in(0,1), g \in L_{2}\left(0, T ; L_{6 / 5}(\Omega)\right) \cap L_{2,-\left(1+\varepsilon_{*}\right)}\left(\Omega^{T}\right), h(0) \in$ $H^{1}(\Omega) \cap H_{-\left(1+\varepsilon_{*}\right)}^{1}(\Omega), 0<\varepsilon_{*}<\delta$ and let $\varepsilon_{*}$ be sufficiently small. Then solutions of (2.11) satisfy

$$
\begin{aligned}
& \|h\|_{2,-\left(1+\varepsilon_{*}\right), \Omega^{T}}+\|q\|_{L_{2}\left(0, T ; H_{-\left(1+\varepsilon_{*}\right)}^{2}(\Omega)\right)} \\
& \leq \varphi\left(\|v\|_{\mathfrak{N}\left(\Omega^{T}\right)}\right)\left(\mathbf{|} g \mathbf{|}_{6 / 5,2, \Omega^{T}}+|h(0)|_{2, \Omega}\right) \\
& \quad+c\left(|g|_{2,-\left(1+\varepsilon_{*}\right), \Omega^{T}}+\|h(0)\|_{1,-\left(1+\varepsilon_{*}\right), \Omega}\right)
\end{aligned}
$$

where $\varphi$ is an increasing positive function and $c$ does not depend on $T$.

Proof. For solutions to problem (2.11) we have (see [8])

$$
\begin{aligned}
& \|h\|_{2,-\left(1+\varepsilon_{*}\right), \Omega^{T}}+\|q\|_{L_{2}\left(0, T ; H_{-\left(1+\varepsilon_{*}\right)}^{1}(\Omega)\right)} \\
& \leq c\left(|G|_{2,-\left(1+\varepsilon_{*}\right), \Omega^{T}}+\|h(0)\|_{1,-\left(1+\varepsilon_{*}\right), \Omega}\right) .
\end{aligned}
$$

By some interpolation inequalities we obtain

$$
\begin{gathered}
\left(\int_{0}^{T}|v \cdot \nabla h|_{2,-1-\varepsilon_{*}, \Omega}^{2} d t\right)^{1 / 2} \leq\left(\int_{0}^{T}|v|_{4,-\delta, \Omega}^{2}|\nabla h|_{4, \delta-1-\varepsilon_{*}, \Omega}^{2} d t\right)^{1 / 2} \\
\leq \varepsilon_{1}\|h\|_{2, \delta-1-\varepsilon_{*}, \Omega^{T}}+\varphi_{1}\left(1 / \varepsilon_{1},|v|_{4, \infty,-\delta, \Omega^{T}}\right)|h|_{2, \delta-1-\varepsilon_{*}, \Omega^{T}}
\end{gathered}
$$

where $\varphi_{1}$ is an increasing positive function.

By the Hardy inequality and for $\delta>\varepsilon_{*}$ we have

$$
|h|_{2, \delta-1-\varepsilon_{*}, \Omega^{T}} \leq c|\nabla h|_{2, \delta-\varepsilon_{*}, \Omega^{T}} \leq c|\nabla h|_{2, \Omega^{T}},
$$

for a bounded domain. Similarly,

$$
\begin{aligned}
& \left(\int_{0}^{T}|h \cdot \nabla v|_{2,-1-\varepsilon_{*}, \Omega}^{2} d t\right)^{1 / 2} \leq\left(\int_{0}^{T}|\nabla v|_{2,-\delta, \Omega}^{2}|h|_{\infty, \delta-1-\varepsilon_{*}, \Omega}^{2} d t\right)^{1 / 2} \\
& \leq \varepsilon_{2}\|h\|_{2, \delta-1-\varepsilon_{*}, \Omega^{T}}+\varphi_{2}\left(1 / \varepsilon_{2},|\nabla v|_{2, \infty,-\delta, \Omega^{T}}\right)|h|_{2, \delta-1-\varepsilon_{*}, \Omega^{T}},
\end{aligned}
$$

where the last norm is estimated by $(2.17)$ and $\varphi_{2}$ is an increasing positive function.

Assuming $\varepsilon_{1}, \varepsilon_{2}$ sufficiently small we obtain from (2.13), (2.16), (2.18) inequality (2.14). This ends the proof.

3. Estimates. In this section we obtain a global a priori estimate for solutions to (1.1). From Lemma 4.3.1 from [7] we have

LEMma 3.1. Assume that $h \in H_{-1}^{2,1}\left(\Omega^{T}\right), u \in L_{2}\left(0, T ; L_{4,-\frac{3}{4}-\varepsilon_{0}}(\Omega)\right), w \in L_{\infty}\left(0, T ; H^{1}(\Omega)\right)$, $f_{\varphi} \in L_{2,-1}\left(\Omega^{T}\right), \chi(0) \in L_{2,-1}(\Omega)$ and $\varepsilon_{0}>0$ is any small number. Then solutions to problem (2.5) satisfy

$$
\begin{gathered}
|\chi(t)|_{2,-1, \Omega}^{2}+\nu \int_{0}^{t}\left\|\chi\left(t^{\prime}\right) / r\right\|_{1, \Omega}^{2} d t^{\prime} \leq c \exp \left(c|h|_{3,2,-1, \Omega^{t}}^{2}\right) \cdot \\
{\left[\left(1+\sup _{t^{\prime} \leq t}\left\|w\left(t^{\prime}\right)\right\|_{1,0, \Omega}^{2}\right)\|h\|_{L_{2}\left(0, t ; H_{-1}^{2}(\Omega)\right)}^{2}\right.} \\
\left.+\sup _{t^{\prime} \leq t}\left\|w\left(t^{\prime}\right)\right\|_{1,0, \Omega}^{2} \int_{0}^{t}\left|u\left(t^{\prime}\right)\right|_{4,-3 / 4-\varepsilon_{0}, \Omega}^{2} d t^{\prime}+\left|F_{\varphi}\right|_{2,-1, \Omega^{t}}^{2}+|\chi(0)|_{2,-1, \Omega}^{2}\right], \quad t \leq T,
\end{gathered}
$$

where the constant $c$ does not depend on $T$. 
To estimate the second factor from the second term on the r.h.s. of (3.1) we need LEMma 3.2. Assume that $v \in L_{2}\left(0, T ; L_{\infty}(\Omega)\right), w \in L_{\infty}\left(0, T ; H_{0}^{1}(\Omega)\right) \cap L_{2}\left(\Omega^{T}\right), v_{, z} \in$ $L_{2}\left(0, T ; L_{\frac{3}{1-\mu}}(\Omega)\right), f_{\varphi} \in L_{2,-\mu}\left(\Omega^{T}\right), q \in L_{2,-(1+\mu)}\left(\Omega^{T}\right), h \in L_{2,-(1+\mu)}\left(\Omega^{T}\right), u(0) \in$ $L_{2,-\mu}(\Omega)$ and $\mu \in(0,1)$. Then solutions of (2.10) satisfy the inequality

$$
\begin{gathered}
|u(t)|_{2,-\mu, \Omega}^{2}+|\nabla u|_{2,-\mu, \Omega^{t}}^{2}+|u|_{2,-(1+\mu), \Omega^{t}}^{2} \\
\leq c \exp \left(c t+c|v|_{\infty, 2, \Omega^{t}}^{2}\right)\left[\sup _{t}\|w\|_{1,0, \Omega}^{2}|v, z|_{\frac{3}{1-\mu}, 2, \Omega^{t}}^{2}\right. \\
\left.+\left|f_{\varphi}\right|_{2,-\mu, \Omega^{t}}^{2}+|q|_{2,-(1+\mu), \Omega^{t}}^{2}+|h|_{2,-(2+\mu), \Omega^{t}}^{2}+|u(0)|_{2,-\mu, \Omega}^{2}\right],
\end{gathered}
$$

where $t \leq T$ and $c$ does not depend on $T$.

Proof. Multiplying $(2.10)_{1}$ by $u r^{-2 \mu}$ and integrating over $\Omega_{\varepsilon_{*}}=\left\{x \in \Omega: 0<\varepsilon_{*}<r\right\}$ and using that $\left.u\right|_{r=\varepsilon_{*}}=0$ yields

$$
\begin{aligned}
& \frac{1}{2} \frac{d}{d t}|u|_{2,-\mu, \Omega_{\varepsilon_{*}}}^{2}+\nu\left(1-\varepsilon_{0}\right)|\nabla u|_{2,-\mu, \Omega_{\varepsilon_{*}}}^{2}+\nu\left(1-\frac{\mu^{2}}{\varepsilon_{0}}\right)|u|_{2,-(1+\mu), \Omega_{\varepsilon_{*}}}^{2} \\
& \leq-\int_{\Omega_{\varepsilon_{*}}}\left(v \cdot \nabla u \frac{u}{r^{2 \mu}}+\frac{v_{r}}{r} \frac{u^{2}}{r^{2 \mu}}\right) d x-\int_{\Omega_{\varepsilon_{*}}}\left(v_{, z} \cdot \nabla w+\frac{v_{r, z}}{r} w\right) \frac{u}{r^{2 \mu}} d x \\
& \quad+\nu \int_{\Omega_{\varepsilon_{*}}} \operatorname{div}\left(\nabla u u r^{-2 \mu}\right) d x+\int_{\Omega_{\varepsilon_{*}}} f_{\varphi, z} \frac{u}{r^{2 \mu}} d x+\int_{\Omega_{\varepsilon_{*}}}\left(\frac{1}{r} q, z+\frac{2 \nu}{r^{2}} h_{r, z}\right) \frac{u}{r^{2 \mu}} d x .
\end{aligned}
$$

The first term on the r.h.s. equals

$$
-(1+\mu) \int_{\Omega_{\varepsilon_{*}}} \frac{v_{r}}{r} \frac{u^{2}}{r^{2 \mu}} d x \equiv I_{1},
$$

where

$$
\left|I_{1}\right| \leq \varepsilon_{1}|u|_{2,-(1+\mu), \Omega_{\varepsilon_{*}}}^{2}+c\left(1 / \varepsilon_{1}\right)|v|_{\infty, \Omega_{\varepsilon_{*}}}^{2}|u|_{2,-\mu, \Omega_{\varepsilon_{*}}}^{2} .
$$

The second term on the r.h.s. of (3.3) is estimated by

$$
\varepsilon_{2}|u|_{\frac{2 s}{s-2},-2 \mu, \Omega_{\varepsilon_{*}}}^{2}+c\left(1 / \varepsilon_{2}\right)\left|v_{, z}\right|_{s, \Omega_{\varepsilon_{*}}}^{2}\|w\|_{1,0, \Omega_{\varepsilon_{*}}}^{2},
$$

where $\frac{3}{s}=1-\mu, s>2$.

By (2.1) we have

$$
|u|_{\frac{2 s}{s-2},-2 \mu, \Omega} \leq c\|u\|_{1,-\mu, \Omega} .
$$

The last two terms on the r.h.s. of (3.3) equal

$$
-\int_{\Omega_{\varepsilon_{*}}}\left(f_{\varphi}+\frac{q}{r}+\frac{2 \nu}{r^{2}} h_{r}\right) \frac{u_{, z}}{r^{2 \mu}} d x \equiv I_{2},
$$

so

$$
\left|I_{2}\right| \leq \varepsilon_{3}\left|u_{, z}\right|_{2,-\mu, \Omega_{\varepsilon_{*}}}^{2}+c\left(1 / \varepsilon_{3}\right)\left(\left|f_{\varphi}\right|_{2,-\mu, \Omega_{\varepsilon_{*}}}^{2}+|q|_{2,-(1+\mu), \Omega_{\varepsilon_{*}}}^{2}+|h|_{2,-(2+\mu), \Omega_{\varepsilon_{*}}}^{2}\right) .
$$

In view of the boundary conditions $(2.10)_{2,3}$ the third term on the r.h.s. of (3.3) equals

$$
\frac{\nu}{R^{1+2 \mu}} \int_{S_{1}} u^{2} d S_{1} \equiv I_{3}
$$

By the extension theorem we have

$$
I_{3} \leq \varepsilon_{4}|\nabla u|_{2, \Omega_{*}}^{2}+c\left(1 / \varepsilon_{4}\right)|u|_{2, \Omega_{*}}^{2} .
$$


Using the above estimates in (3.3) yields

$$
\begin{gathered}
\frac{1}{2} \frac{d}{d t}|u|_{2,-\mu, \Omega_{\varepsilon_{*}}}^{2}+\nu\left(1-\varepsilon_{0}-\varepsilon_{2}-\varepsilon_{3}-\varepsilon_{4}\right)|\nabla u|_{2,-\mu, \Omega_{\varepsilon_{*}}}^{2} \\
+\nu\left(1-\frac{\mu^{2}}{\varepsilon_{0}}-\varepsilon_{1}-\varepsilon_{2}-\varepsilon_{3}\right)|u|_{2,-(1+\mu), \Omega_{\varepsilon_{*}}}^{2} \\
\leq c|v|_{\infty, \Omega_{\varepsilon_{*}}}^{2}|u|_{2,-\mu, \Omega_{\varepsilon_{*}}}^{2}+c\left|v_{, z}\right|_{\frac{3}{1-\mu}, \Omega_{\varepsilon_{*}}}^{2}\|w\|_{1,0, \Omega_{\varepsilon_{*}}}^{2} \\
+c\left(\left|f_{\varphi}\right|_{2,-\mu, \Omega_{\varepsilon_{*}}}^{2}+|q|_{2,-(1+\mu), \Omega_{\varepsilon_{*}}}^{2}+|h|_{2,-(2+\mu), \Omega_{\varepsilon_{*}}}^{2}\right)+c|u|_{2, \Omega_{\varepsilon_{*}}}^{2} .
\end{gathered}
$$

Assuming that $\varepsilon_{0}-\varepsilon_{4}$ are such that the coefficients near the last two terms on the l.h.s. of (3.5) are positive, integrating the result with respect to time and passing with $\varepsilon_{*}$ to 0 we obtain (3.2). This ends the proof.

Let us consider problem (2.6).

Lemma 3.3. Let $v \in L_{2}\left(0, T ; L_{\infty}((\Omega)), q \in L_{2}\left(\Omega^{T}\right), h \in L_{2}\left(0, T ; L_{2,-1}(\Omega)\right), f_{\varphi} \in\right.$ $L_{2}\left(\Omega^{T}\right), w(0) \in L_{2}(\Omega)$. Then solutions of (2.6) satisfy

$$
\begin{gathered}
|w(t)|_{2, \Omega}^{2}+\nu \int_{0}^{t}\left\|w\left(t^{\prime}\right)\right\|_{1,0, \Omega}^{2} d t^{\prime} \leq c \exp \left(c t+c|v|_{\infty, 2, \Omega^{t}}^{2}\right) \\
\quad \times\left[|q|_{2, \Omega^{t}}^{2}+|h|_{2,-1, \Omega^{t}}^{2}+\left|f_{\varphi}\right|_{2, \Omega^{t}}^{2}+|w(0)|_{2, \Omega}^{2}\right], \quad t \leq T,
\end{gathered}
$$

where $c$ does not depend on $t$.

Proof. Multiplying $(2.6)_{1}$ by $w$ and integrating over $\Omega_{\varepsilon_{*}}$ (for the definition see the proof of Lemma 3.2) yields

$$
\begin{aligned}
& \frac{1}{2} \frac{d}{d t}|w|_{2, \Omega_{\varepsilon_{*}}}^{2}+\nu|\nabla w|_{2, \Omega_{\varepsilon_{*}}}^{2}+\nu|w|_{2,-1, \Omega_{\varepsilon_{*}}}^{2}-\frac{\nu}{R}|w|_{2, S_{1}}^{2} \\
& =-\int_{\Omega_{\varepsilon_{*}}} \frac{v_{r}}{r} w^{2} d x+\int_{\Omega_{\varepsilon_{*}}}\left(\frac{1}{r} q+\frac{2 \nu}{r^{2}} h_{r}+f_{\varphi}\right) w d x .
\end{aligned}
$$

The last term on the l.h.s. is estimated by

$$
\varepsilon_{1}|\nabla w|_{2, \Omega_{\varepsilon_{*}}}^{2}+c\left(1 / \varepsilon_{1}\right)|w|_{2, \Omega_{\varepsilon_{*}}}^{2} .
$$

By the Young and the Hölder inequalities we estimate the first term on the r.h.s. of (3.7) by

$$
\varepsilon_{2}|w|_{2,-1, \Omega_{\varepsilon_{*}}}^{2}+c\left(1 / \varepsilon_{2}\right)|v|_{\infty, \Omega_{*}}^{2}|w|_{2, \Omega_{\varepsilon_{*}}}^{2} .
$$

Finally, the last term on the r.h.s. of (3.7) is estimated by

$$
\varepsilon_{3}|w|_{2,-1, \Omega_{\varepsilon_{*}}}^{2}+c\left(1 / \varepsilon_{3}\right)\left(|q|_{2, \Omega_{*}}^{2}+|h|_{2,-1, \Omega_{\varepsilon_{*}}}^{2}\left|f_{\varphi}\right|_{2, \Omega_{\varepsilon_{*}}}^{2}\right) .
$$

Using the above estimates in (3.7) with sufficiently small $\varepsilon_{1}-\varepsilon_{3}$ we obtain

$$
\begin{gathered}
\frac{d}{d t}|w|_{2, \Omega_{\varepsilon_{*}}}^{2}+\nu|\nabla w|_{2, \Omega_{\varepsilon_{*}}}^{2}+\nu|w|_{2,-1, \Omega_{\varepsilon_{*}}}^{2} \\
\leq c|w|_{2, \Omega_{\varepsilon_{*}}}^{2}+c|v|_{\infty, \Omega_{\varepsilon_{*}}}^{2}|w|_{2, \Omega_{\varepsilon_{*}}}^{2}+c\left(|q|_{2, \Omega_{\varepsilon_{*}}}^{2}+|h|_{2,-1, \Omega_{\varepsilon_{*}}}^{2}+\left|f_{\varphi}\right|_{2, \Omega_{\varepsilon_{*} *}}^{2}\right) .
\end{gathered}
$$

Integrating (3.8) with respect to time and passing with $\varepsilon_{*}$ to 0 yields (3.6). This concludes the proof. 
Formula (5.3.22) from [7] yields

$$
\begin{aligned}
\|w(t)\|_{1,0, \Omega}^{2} \leq & c \exp \left(c|v|_{\infty, 2, \Omega^{t}}^{2}\right)\left[\left(1+|v|_{\infty, 4, \Omega^{t}}^{4}\right) \sup _{t}|w(t)|_{2,1, \Omega}^{2}\right. \\
& \left.+|w|_{2, \Omega^{t}}^{2}+|q|_{2, \Omega^{t}}^{2}+|h|_{2,-1, \Omega^{t}}^{2}+\left|f_{\varphi}\right|_{2,1, \Omega^{t}}^{2}+e^{-t}\|w(0)\|_{1,0, \Omega}^{2}\right]
\end{aligned}
$$

where Lemma 6.3.5 from [7] gives

$$
\begin{aligned}
|w(t)|_{2,1, \Omega} \leq & |w(0)|_{2,1, \Omega}+c \int_{0}^{t}\left(\left|q\left(t^{\prime}\right)\right|_{2, \Omega}+\left|h\left(t^{\prime}\right)\right|_{2,-1, \Omega}\right. \\
& \left.+\left|f_{\varphi}\left(t^{\prime}\right)\right|_{2,1, \Omega}\right) d t^{\prime}, \quad t \leq T .
\end{aligned}
$$

Finally we obtain an estimate for solutions to problems (2.8), (2.9):

LEMMA 3.4. Assume that $\alpha^{\prime}=\left(\alpha_{r}, \alpha_{z}\right), v \in L_{2}\left(0, T ; W_{3}^{1}(\Omega)\right) \cap L_{\infty}\left(0, T ; L_{3}(\Omega)\right), w \in$ $W_{2}^{1,1 / 2}\left(\Omega^{T}\right) \cap L_{\infty}\left(0, T ; H^{s}(\Omega)\right), s>1 / 2, h \in L_{\infty}\left(0, T ; L_{3 / 2}(\Omega)\right) \cap L_{2}\left(0, T ; H_{-1}^{1}(\Omega)\right)$, $u \in L_{2}\left(0, T ; H^{1}(\Omega)\right), \chi / r \in L_{2}\left(0, T ; L_{6}(\Omega)\right), F^{\prime}=\left(F_{r}, F_{z}\right) \in L_{2}\left(0, T ; L_{6 / 5}(\Omega)\right), \alpha^{\prime}(0) \in$ $L_{2}(\Omega)$.

Then solutions of problems (2.8) and (2.9) satisfy

$$
\begin{gathered}
\left|\alpha^{\prime}(t)\right|_{2, \Omega}^{2}+\nu \int_{0}^{t}\left\|\alpha^{\prime}\left(t^{\prime}\right)\right\|_{1, \Omega}^{2} d t^{\prime} \\
\leq c \exp \left(\left|v_{, x}\right|_{3,2, \Omega^{t}}^{2}\right)\left[|v|_{3, \infty, \Omega^{t}}^{2}\|w\|_{W_{2}^{1,1 / 2}\left(\Omega^{t}\right)}^{2}+\left|v_{, x}\right|_{3,2, \Omega^{t}}^{2}\|w\|_{L_{\infty}\left(0, t ; H^{s}(\Omega)\right)}^{2}\right. \\
+\left.|h|_{3 / 2, \infty, \Omega^{t}}^{2}|\chi| r\right|_{6,2, \Omega^{t}} ^{2}+\int_{0}^{t}\left(\left\|h\left(t^{\prime}\right)\right\|_{1,-1, \Omega}^{2}+\left\|u\left(t^{\prime}\right)\right\|_{1, \Omega}^{2}\right) d t^{\prime} \\
\left.+\left|F^{\prime}\right|_{6 / 5,2, \Omega^{t}}^{2}+\left|\alpha^{\prime}(0)\right|_{2, \Omega}^{2}\right]+\|w\|_{L_{\infty}\left(0, t ; H^{s}(\Omega)\right)}+\|w\|_{W_{2}^{1,1 / 2}\left(\Omega^{t}\right)}
\end{gathered}
$$

where $t \leq T$ and $s-\frac{1}{2}$ is an arbitrary small positive number.

Proof. Multiplying $(2.8)_{1}$ by $\alpha_{r}$ and integrating over $\Omega$ yields

$$
\begin{gathered}
\frac{1}{2} \frac{d}{d t}\left|\alpha_{r}\right|_{2, \Omega}^{2}+\nu\left\|\alpha_{r}\right\|_{1,0, \Omega}^{2} \leq\left|\int_{S_{1}}\left(\frac{1}{R^{3}} h_{z}+\frac{1}{R} u\right) \alpha_{r} d S_{1}\right| \\
+\left|\int_{\Omega} \frac{\chi}{r} h_{r} \alpha_{r} d x\right|+\int_{\Omega}\left|v_{x}\right|\left(\alpha_{r}^{2}+\alpha_{z}^{2}\right) d x+2 \nu \int_{\Omega} \frac{1}{r^{2}}\left|h_{, x}\right|\left|\alpha_{r}\right| d x \\
+\left|\int_{\Omega} F_{r} \alpha_{r} d x\right| .
\end{gathered}
$$

The first term on the r.h.s. is estimated by

$$
c\left|\alpha_{r}\right|_{4, S_{1}}\left(|h|_{4 / 3, S_{1}}+|u|_{4 / 3, S_{1}}\right) \leq \varepsilon_{1}\left\|\alpha_{r}\right\|_{1, \Omega}^{2}+c\left(1 / \varepsilon_{1}\right)\left(\|h\|_{1, \Omega}^{2}+\|u\|_{1, \Omega}^{2}\right),
$$

the second by

$$
\varepsilon_{2}\left|\alpha_{r}\right|_{6, \Omega}^{2}+c\left(1 / \varepsilon_{2}\right)|\chi / r|_{6, \Omega}^{2}|h|_{3 / 2, \Omega}^{2},
$$

the third by

$$
\varepsilon_{3}\left(\left|\alpha_{r}\right|_{6, \Omega}^{2}+\left|\alpha_{z}\right|_{6, \Omega}^{2}\right)+c\left(1 / \varepsilon_{3}\right)\left|v_{, x}\right|_{3, \Omega}^{2}\left(\left|\alpha_{r}\right|_{2, \Omega}^{2}+\left|\alpha_{z}\right|_{2, \Omega}^{2}\right),
$$

the fourth by

$$
\varepsilon_{4}\left|\alpha_{r}\right|_{2,-1, \Omega}^{2}+c\left(1 / \varepsilon_{4}\right)\left|h_{, x}\right|_{2,-1, \Omega}^{2}
$$


and the last by

$$
\varepsilon_{5}\left|\alpha_{r}\right|_{6, \Omega}^{2}+c\left(1 / \varepsilon_{5}\right)\left|F_{r}\right|_{6 / 5, \Omega}^{2}
$$

Hence, for sufficiently small $\varepsilon_{1}-\varepsilon_{5}$ we have

$$
\begin{gathered}
\frac{d}{d t}\left|\alpha_{r}\right|_{2, \Omega}^{2}+\nu\left\|\alpha_{r}\right\|_{1,0, \Omega}^{2} \leq c\left(\|h\|_{1, \Omega}^{2}+\|u\|_{1, \Omega}^{2}+|h|_{3 / 2, \Omega}^{2}|\chi / r|_{6, \Omega}^{2}\right. \\
\left.+\left|v_{, x}\right|_{3, \Omega}^{2}\left(\left|\alpha_{r}\right|_{2, \Omega}^{2}+\left|\alpha_{z}\right|_{2, \Omega}^{2}\right)+\left|h_{, x}\right|_{2,-1, \Omega}^{2}+\left|F_{r}\right|_{6 / 5, \Omega}^{2}\right) .
\end{gathered}
$$

To examine problem (2.9) we introduce a function $\beta$ such that

$$
\begin{array}{cl}
\beta, t-\nu \Delta \beta=0 & \text { in } \Omega^{T}, \\
\left.\beta\right|_{S_{1}}=\frac{2}{R} w & \text { on } S_{1}^{T}, \\
\left.\beta{ }_{, z}\right|_{S_{2}}=0 & \text { on } S_{2}^{T}, \\
\left.\beta\right|_{t=0}=0 & \text { in } \Omega .
\end{array}
$$

Introducing the new function

$$
\alpha_{z}^{\prime}=\alpha_{z}-\beta,
$$

we see that it is a solution to the problem

$$
\begin{gathered}
\alpha_{z, t}^{\prime}-\nu \Delta \alpha_{z}^{\prime}=-v \cdot \nabla \alpha_{z}+\left(\alpha_{r} v_{z, r}+\alpha_{z} v_{z, z}\right)+\frac{\chi}{r} h_{z}+F_{z} \quad \text { in } \Omega^{T}, \\
\left.\alpha_{z}^{\prime}\right|_{S_{1}}=0,\left.\quad \alpha_{z, z}^{\prime}\right|_{S_{2}}=0,\left.\quad \alpha_{z}^{\prime}\right|_{t=0}=\alpha_{z}(0) .
\end{gathered}
$$

Multiplying $(3.16)_{1}$ by $\alpha_{z}^{\prime}$ and integrating over $\Omega$ we obtain

$$
\begin{gathered}
\frac{1}{2} \frac{d}{d t}\left|\alpha_{z}^{\prime}\right|_{2, \Omega}^{2}+\nu\left\|\alpha_{z}^{\prime}\right\|_{1, \Omega}^{2}=-\int_{\Omega} v \cdot \nabla \alpha_{z} \alpha_{z}^{\prime} d x \\
+\int_{\Omega}\left(\alpha_{r} v_{z, r}+\alpha_{z} v_{z, z}\right) \alpha_{z}^{\prime} d x+\int_{\Omega} \frac{\chi}{r} h_{z} \alpha_{z}^{\prime} d x+\int_{\Omega} F_{z} \alpha_{z}^{\prime} d x .
\end{gathered}
$$

The first term on the r.h.s. equals

$$
-\int_{\Omega} v \cdot \nabla\left(\alpha_{z}^{\prime}+\beta\right) \cdot \alpha_{z}^{\prime} d x=-\int_{\Omega} v \cdot \nabla \beta \alpha_{z}^{\prime} d x \equiv I_{1},
$$

where

$$
\left|I_{1}\right| \leq \varepsilon_{1}\left|\alpha_{z}^{\prime}\right|_{6, \Omega}^{2}+c\left(1 / \varepsilon_{1}\right)|v|_{3, \Omega}^{2}|\nabla \beta|_{2, \Omega}^{2} .
$$

The second term on the r.h.s. of (3.17) is estimated by

$$
\varepsilon_{2}\left|\alpha_{z}^{\prime}\right|_{6, \Omega}^{2}+c\left(1 / \varepsilon_{2}\right)\left|v_{, x}\right|_{3, \Omega}^{2}\left(\left|\alpha_{r}\right|_{2, \Omega}^{2}+\left|\alpha_{z}\right|_{2, \Omega}^{2}\right),
$$

the third term by

$$
\varepsilon_{3}\left|\alpha_{z}^{\prime}\right|_{6, \Omega}^{2}+c\left(1 / \varepsilon_{3}\right)|\chi / r|_{6, \Omega}^{2}|h|_{3 / 2, \Omega}^{2},
$$

and finally the last term by

$$
\varepsilon_{4}\left|\alpha_{z}^{\prime}\right|_{6, \Omega}^{2}+c\left(1 / \varepsilon_{4}\right)\left|F_{z}\right|_{6 / 5, \Omega}^{2}
$$

In view of the above estimates and for sufficiently small $\varepsilon_{1}$ through $\varepsilon_{4}$ we obtain from (3.17) the inequality

$$
\begin{gathered}
\frac{d}{d t}\left|\alpha_{z}^{\prime}\right|_{2, \Omega}^{2}+\nu\left\|\alpha_{z}^{\prime}\right\|_{1, \Omega}^{2} \leq c\left[|v|_{3, \Omega}^{2}|\nabla \beta|_{2, \Omega}^{2}\right. \\
\left.+\left|v_{, x}\right|_{3, \Omega}^{2}\left(\left|\alpha_{r}\right|_{2, \Omega}^{2}+\left|\alpha_{z}\right|_{2, \Omega}^{2}\right)+\left.|\chi| r\right|_{6, \Omega} ^{2}|h|_{3 / 2, \Omega}^{2}+\left|F_{z}\right|_{6 / 5, \Omega}^{2}\right] .
\end{gathered}
$$


Using (3.15) in (3.13),

$$
\begin{gathered}
\frac{d}{d t}\left|\alpha_{r}\right|_{2, \Omega}^{2}+\nu\left\|\alpha_{r}\right\|_{1,0, \Omega}^{2} \leq c\left|v_{, x}\right|_{3, \Omega}^{2}\left(\left|\alpha_{r}\right|_{2, \Omega}^{2}+\left|\alpha_{z}^{\prime}\right|_{2, \Omega}^{2}\right) \\
+c\left|v_{, x}\right|_{3, \Omega}^{2}|\beta|_{2, \Omega}^{2}+c\left(\|h\|_{1, \Omega}^{2}+\|u\|_{1, \Omega}^{2}\right)+c|h|_{3 / 2, \Omega}^{2}|\chi / r|_{6, \Omega}^{2} \\
+c\left|h_{, x}\right|_{2,-1, \Omega}^{2}+c\left|F_{r}\right|_{6 / 5, \Omega}^{2}
\end{gathered}
$$

and in (3.18),

$$
\begin{gathered}
\frac{d}{d t}\left|\alpha_{z}^{\prime}\right|_{2, \Omega}^{2}+\nu\left\|\alpha_{z}^{\prime}\right\|_{1, \Omega}^{2} \leq c\left|v_{, x}\right|_{3, \Omega}^{2}\left(\left|\alpha_{r}\right|_{2, \Omega}^{2}+\left|\alpha_{z}^{\prime}\right|_{2, \Omega}^{2}\right) \\
+c\left(|v|_{3, \Omega}^{2}|\nabla \beta|_{2, \Omega}^{2}+\left|v_{, x}\right|_{3, \Omega}^{2}|\beta|_{2, \Omega}^{2}\right)+c|h|_{3 / 2, \Omega}^{2}|\chi / r|_{6, \Omega}^{2} \\
+c\left|F_{z}\right|_{6 / 5, \Omega}^{2} .
\end{gathered}
$$

Adding (3.19) and (3.20) and integrating the result with respect to time yields

$$
\begin{gathered}
\left|\alpha_{r}(t)\right|_{2, \Omega}^{2}+\left|\alpha_{z}^{\prime}(t)\right|_{2, \Omega}^{2}+\nu \int_{0}^{t}\left(\left\|\alpha_{r}\left(t^{\prime}\right)\right\|_{1,0, \Omega}^{2}+\left\|\alpha_{z}^{\prime}\left(t^{\prime}\right)\right\|_{1, \Omega}^{2}\right) d t^{\prime} \\
\leq c \exp \left(c\left|v_{, x}\right|_{3,2, \Omega^{t}}^{2}\right)\left[|v|_{3, \infty, \Omega^{t}}^{2}|\nabla \beta|_{2, \Omega^{t}}^{2}+\left|v_{, x}\right|_{3,2, \Omega^{t}}^{2}|\beta|_{2, \infty, \Omega^{t}}^{2}\right. \\
+|h|_{3 / 2, \infty, \Omega^{t}}^{2}|\chi / r|_{6,2, \Omega^{t}}^{2}+\int_{0}^{t}\left(\left\|h\left(t^{\prime}\right)\right\|_{1, \Omega}^{2}+\left\|u\left(t^{\prime}\right)\right\|_{1, \Omega}^{2}+\left|h_{, x}\left(t^{\prime}\right)\right|_{2,-1, \Omega}^{2}\right) d t^{\prime} \\
\left.+\left|F^{\prime}\right|_{6 / 5,2, \Omega^{t}}^{2}+\left|\alpha^{\prime}(0)\right|_{2, \Omega}^{2}\right],
\end{gathered}
$$

where $F^{\prime}=\left(F_{r}, F_{z}\right), \alpha^{\prime}=\left(\alpha_{r}, \alpha_{z}\right)$.

Using again (3.15) we obtain

$$
\begin{gathered}
\left|\alpha^{\prime}(t)\right|_{2, \Omega}^{2}+\nu \int_{0}^{t}\left\|\alpha^{\prime}\left(t^{\prime}\right)\right\|_{1, \Omega}^{2} d t^{\prime} \leq c \exp \left(c\left|v_{, x}\right|_{3,2, \Omega^{t}}^{2}\right) \\
\cdot\left[|v|_{3, \infty, \Omega^{t}}^{2}|\nabla \beta|_{2, \Omega^{t}}^{2}+\left|v_{, x}\right|_{3,2, \Omega^{t}}^{2}|\beta|_{2, \infty, \Omega^{t}}^{2}\right. \\
+|h|_{3 / 2, \infty, \Omega^{t}}^{2}|\chi / r|_{6,2, \Omega^{t}}^{2}+\int_{0}^{t}\left(\left\|h\left(t^{\prime}\right)\right\|_{1,-1, \Omega}^{2}+\left\|u\left(t^{\prime}\right)\right\|_{1, \Omega}^{2}\right) d t^{\prime} \\
\left.+\left|F^{\prime}\right|_{6 / 5,2, \Omega^{t}}^{2}+\left|\alpha^{\prime}(0)\right|_{2, \Omega}^{2}\right]+|\beta|_{2, \infty, \Omega^{t}}^{2}+\nu \int_{0}^{t}\left\|\beta\left(t^{\prime}\right)\right\|_{1, \Omega}^{2} d t^{\prime} .
\end{gathered}
$$

By the potential theory techniques we have (see Lemmas 6.1, 6.2)

$$
\begin{array}{r}
|\beta|_{2, \infty, S^{t}} \leq c|w|_{2, \infty, S^{t}} \leq c \sup _{t}\|w\|_{s, \Omega}, \quad s>\frac{1}{2}, \\
\left(\int_{0}^{t}\left\|\beta\left(t^{\prime}\right)\right\|_{1, \Omega}^{2} d t^{\prime}\right)^{1 / 2} \leq c\|w\|_{W_{2}^{1 / 2,1 / 4}\left(S^{t}\right)} \leq c\|w\|_{W_{2}^{1,1 / 2}\left(\Omega^{t}\right)} .
\end{array}
$$

Employing (3.23) in (3.22) yields (3.11). This concludes the proof.

To estimate the second factor of the second term in the square bracket on the r.h.s. of (3.1) we employ (3.2) for $\mu=\frac{1}{2}+\varepsilon_{0}$. Then (3.1) takes the form

$$
|\chi(t)|_{2,-1, \Omega}^{2}+\nu \int_{0}^{t}\left\|\chi\left(t^{\prime}\right) / r\right\|_{1, \Omega}^{2} d t^{\prime} \leq c \exp \left(c|h|_{3,2,-1, \Omega^{t}}^{2}\right)
$$




$$
\begin{gathered}
\times\left[\left(1+\|w\|_{L_{\infty}\left(0, t ; H_{0}^{1}(\Omega)\right)}^{2}\right)\|h\|_{L_{2}\left(0, t ; H_{-1}^{2}(\Omega)\right)}^{2}+\|w\|_{L_{\infty}\left(0, t ; H_{0}^{1}(\Omega)\right)}^{2} \exp \left(c t+c|v|_{\infty, 2, \Omega^{t}}^{2}\right)\right. \\
\times\left(\|w\|_{L_{\infty}\left(0, t ; H_{0}^{1}(\Omega)\right)}^{2}|v, z|_{\frac{6}{1-2 \varepsilon_{0}}, 2, \Omega^{t}}^{2}\right. \\
\left.+\left|f_{\varphi}\right|_{2,-\frac{1}{2}-\varepsilon_{0}, \Omega^{t}}^{2}+|q|_{2,-\left(\frac{3}{2}+\varepsilon_{0}\right), \Omega^{t}}^{2}+|h|_{2,-\left(\frac{5}{2}+\varepsilon_{0}\right), \Omega^{t}}^{2}+|u(0)|_{2,-\left(\frac{1}{2}+\varepsilon_{0}\right), \Omega}^{2}\right) \\
\left.+\left|F_{\varphi}\right|_{2,-1, \Omega^{t}}^{2}+|\chi(0)|_{2,-1, \Omega^{t}}^{2}\right], \quad t \leq T .
\end{gathered}
$$

4. Estimate of a fixed point. First we obtain an inequality implying an estimate for a fixed point of transformation (1.6).

LEMMA 4.1. Let

$$
\begin{gathered}
Z_{0}(t)=\|v\|_{2,5 / 2, \Omega^{t}} \\
X(t)=|g|_{2,-\left(1+\varepsilon_{*}\right), \Omega^{t}}+\left|f_{\varphi}\right|_{2,-\left(1 / 2+\varepsilon_{0}\right), \Omega^{t}}+\left|F^{\prime}\right|_{6 / 5,2, \Omega^{t}} \\
+\|h(0)\|_{1,-\left(1+\varepsilon_{*}\right), \Omega}+\|w(0)\|_{1,0, \Omega}+\left|\alpha^{\prime}(0)\right|_{2, \Omega}+\mid u\left(\left.0\right|_{2, \Omega}<\infty,\right. \\
Y_{1}(t)=\left|F_{\varphi}\right|_{2,-1, \Omega^{t}}+|\chi(0)|_{2,-1, \Omega^{t}}<\infty \\
Y_{2}(t)=|f|_{5 / 2, \Omega^{t}}+\|v(0)\|_{6 / 5,5 / 2, \Omega}<\infty
\end{gathered}
$$

where $\varepsilon_{*}, \varepsilon_{0}$ are positive arbitrary small numbers and $t \leq T$. Then

$$
Z_{0} \leq \varphi_{1}\left(\varphi(t) Z_{0} X\right)\left[\varphi_{1}\left(t, Z_{0}\right) X\left(1+Y_{1}\right)+Y_{1}\right]^{2}+c_{1} Y_{2}
$$

where $\varphi, \varphi_{1}$ are increasing positive functions.

Proof. Applying Lemmas 2.2 and 2.3 we obtain from (3.6), (3.9), (3.10) the inequality

$$
\|w(t)\|_{1,0, \Omega} \leq \varphi\left(t, Z_{1}(t)\right) X_{1}(t)
$$

where $\varphi$ is an increasing positive function and

$$
\begin{aligned}
& Z_{1}(t)=|v|_{\infty, 4, \Omega^{t}}+\left|v_{, x}\right|_{3,2, \Omega^{t}}+\left|v_{, x}\right|_{2, \infty,-\delta, \Omega^{t}}+|v|_{4, \infty,-\delta, \Omega^{t}}, \\
& X_{1}(t)=|g|_{2,-\left(1+\varepsilon_{*}\right), \Omega^{t}}+\left|f_{\varphi}\right|_{2, \Omega^{t}}+\|h(0)\|_{1,-\left(1+\varepsilon_{*}\right), \Omega}+\|w(0)\|_{1,0, \Omega},
\end{aligned}
$$

and $1>\delta>\varepsilon_{*}>0$.

In virtue of (4.2) and Lemma 2.3 inequality (3.24) assumes the form

$$
\|\chi / r\|_{V_{2}^{0}\left(\Omega^{t}\right)} \leq \varphi\left(t, Z_{2}(t)\right) X_{2}(t)+\varphi\left(Z_{2}(t) X_{2}(t)\right) Y_{1}(t),
$$

where $\varphi$ is an increasing positive function and

$$
\begin{aligned}
Z_{2}(t) & =Z_{1}(t)+\left|v_{, x}\right|_{\frac{6}{1-2 \varepsilon_{0}}, 2, \Omega^{t}}, \\
X_{2}(t) & =X_{1}(t)+\left|f_{\varphi}\right|_{2,-\left(1 / 2+\varepsilon_{0}\right), \Omega^{t}}, \\
Y_{1}(t) & =\left|F_{\varphi}\right|_{2,-1, \Omega^{t}}+|\chi(0)|_{2,-1, \Omega},
\end{aligned}
$$

where $\varepsilon_{0}>0$ is an arbitrary small number. Next, (3.11) yields

$$
\begin{gathered}
\left\|\alpha^{\prime}\right\|_{V_{2}^{0}\left(\Omega^{t}\right)} \leq \varphi\left(Z_{2}\right)\left[\|w\|_{W_{2}^{1,1 / 2}\left(\Omega^{t}\right)}+\|w\|_{L_{\infty}\left(0, t ; H^{1}(\Omega)\right)}+X_{2}\|\chi / r\|_{V_{2}^{0}\left(\Omega^{t}\right)}\right. \\
\left.+X_{1}(t)+\|u\|_{L_{2}\left(0, t ; H^{1}(\Omega)\right)}+X_{3}(t)\right],
\end{gathered}
$$

where

$$
X_{3}(t)=\left|F^{\prime}\right|_{6 / 5,2, \Omega^{t}}+\left|\alpha^{\prime}(0)\right|_{2, \Omega}
$$


In view of (4.2) and (4.4) we obtain

$$
\begin{aligned}
\left\|\alpha^{\prime}\right\|_{V_{2}^{0}\left(\Omega^{t}\right)} \leq & \varphi\left(Z_{2}\right)\left[\varphi(t)\left(X_{4}(t)+X_{2} Y_{1}\right)\right. \\
& \left.+\|w\|_{L_{2}\left(\Omega ; W^{1 / 2}(0, t)\right)}+\|u\|_{L_{2}\left(0, t ; H^{1}(\Omega)\right)}\right],
\end{aligned}
$$

where

$$
X_{4}(t)=X_{2}(t)+X_{3}(t) .
$$

Using (3.2) for $\mu=0,(4.2)$ and Lemma 2.3 we obtain

$$
\|u\|_{V_{2}^{0}\left(\Omega^{t}\right)} \leq \varphi\left(t, Z_{2}\right)\left(X_{2}+|u(0)|_{2, \Omega}\right) .
$$

From Lemma 6.3.3 from [7] we have

$$
\begin{aligned}
\left|w_{, t}\right|_{2, \Omega^{t}} \leq & c\|w(0)\|_{1,0, \Omega}|v|_{\infty, 2, \Omega^{t}}+c\left(|q|_{2,-1, \Omega^{t}}+|h|_{2,-2, \Omega^{t}}+\left|f_{\varphi}\right|_{2, \Omega^{t}}\right) \\
& +c\|w\|_{L_{2}\left(0, t ; H^{1}(\Omega)\right)}+c\|w(0)\|_{1,0, \Omega} .
\end{aligned}
$$

Using (4.2) and Lemma 2.3 in (4.11) implies

$$
\left|w_{, t}\right|_{2, \Omega^{t}} \leq \varphi\left(t, Z_{2}\right) X_{1}(t) .
$$

Employing (4.10) and (4.12) in (4.8) yields

$$
\left\|\alpha^{\prime}\right\|_{V_{2}^{0}\left(\Omega^{t}\right)} \leq \varphi\left(t, Z_{2}\right) X(t)\left(1+Y_{1}\right),
$$

where

$$
X(t)=X_{4}+|u(0)|_{2, \Omega} .
$$

In view of (4.4) and (4.13) we obtain for solutions to problem (2.12) the inequality

$$
|v|_{10, \Omega^{t}}+|\nabla v|_{10 / 3, \Omega^{t}} \leq \varphi(t, Z X) Y_{1}+\varphi(t, Z) X\left(1+Y_{1}\right),
$$

where $Z=Z_{1}+Z_{2}$. In view of (4.15) and the estimate

$$
Z(t) \leq c Z_{0}(t),
$$

we obtain for solutions of problem (1.1) inequality (4.1). This concludes the proof.

Lemma 4.2. Let the assumptions of Lemma 4.1 hold. Let $T<\infty$ be given. Let $A=$ $\sigma\left[\varphi_{1}(0) Y_{1}^{2}+c_{1} Y_{2}\right]$, where $\sigma \geq 2$ and let $\varphi_{1}$ be the function from (4.1). Then for sufficiently small $X$,

$$
Z_{0}(t) \leq A
$$

The proof follows directly from (4.1).

5. Existence. To prove the existence of solutions to problem (1.1) we construct a mapping $\Phi_{2}$ defined by the problem (see [4])

$$
\begin{array}{cc}
v_{, t}-\operatorname{div} \mathbb{T}(v, p)=-\lambda \tilde{v}\left(v^{\prime}\right) \cdot \nabla \tilde{v}\left(v^{\prime}\right)+f & \text { in } \Omega^{T}, \\
\operatorname{div} v=0 & \text { in } \Omega^{T}, \\
v \cdot \bar{n}=0 \quad \bar{n} \cdot \mathbb{T}(v, p) \cdot \bar{\tau}_{\alpha}=0, \quad \alpha=1,2, & \text { on } S^{T}, \\
\left.v\right|_{t=0}=v(0) & \text { in } \Omega,
\end{array}
$$

where $\lambda \in[0,1]$. Hence

$$
v=\Phi_{2}(\tilde{v}, \lambda)
$$


The quantity $\tilde{v}$ is calculated from problem (2.12) where $\alpha$ is determined by (4.4), (4.8) with $Z_{2}=Z_{2}\left(v^{\prime}\right)$ and $v^{\prime}$ is assumed to be a prescribed element of the space

$$
\mathfrak{M}\left(\Omega^{T}\right)=\left\{u: Z_{2}(u)<\infty\right\} .
$$

Then problem (2.12) determines the transformation

$$
\mathfrak{M}\left(\Omega^{T}\right) \ni v^{\prime} \rightarrow \Phi_{1}\left(v^{\prime}\right)=\tilde{v} \in \mathfrak{M}_{0}\left(\Omega^{T}\right)
$$

where

$$
\mathfrak{M}_{0}\left(\Omega^{T}\right)=\left\{u: u \in L_{10}\left(\Omega^{T}\right) \text { and } \nabla u \in L_{\frac{10}{3}}\left(\Omega^{T}\right)\right\}
$$

Hence

$$
\Phi_{2}: \mathfrak{M}_{0}\left(\Omega^{T}\right) \times[0,1] \rightarrow \mathfrak{M}_{*}\left(\Omega^{T}\right)=W_{5 / 2}^{2,1}\left(\Omega^{T}\right)
$$

Defining

$$
\Phi=\Phi_{2} \circ \Phi_{1}
$$

we see that (4.17) is the estimate of a fixed point of $\Phi$.

To prove the existence of solutions to problem (1.1) we apply the Leray-Schauder fixed point theorem. Therefore we have to show the following properties of the mapping

$$
\Phi: \mathfrak{M}\left(\Omega^{T}\right) \times[0,1] \rightarrow \mathfrak{M}_{*}\left(\Omega^{T}\right):
$$

- Compactness.

- Continuity.

- Existence of a unique solution for $\lambda=0$.

LEMMA 5.1. The mapping $\Phi$ is compact and for $\lambda=0$ index $\Phi=1$.

Proof. Compactness follows from the compact imbedding $\mathfrak{M}_{*}\left(\Omega^{T}\right) \subset \mathfrak{M}\left(\Omega^{T}\right)$.

For $\lambda=0$ the unique existence of solutions to the corresponding Stokes system follows from [1]. This ends the proof.

Finally, we show the continuity.

LEMMA 5.2. Let the assumptions of Lemmas 4.1 and 4.2 hold. Then the mapping $\Phi$ : $\mathfrak{M}\left(\Omega^{T}\right) \times[0,1] \rightarrow \mathfrak{M}_{*}\left(\Omega^{T}\right)$ is continuous.

Proof. Assume that functions $v_{s}^{\prime} \in \mathfrak{M}\left(\Omega^{T}\right), s=1,2$, are given. Then we have problems (2.5), (2.6), (2.8), (2.9), (2.10), (2.11) for functions $h_{s}, q_{s}, u_{s}, w_{s}, \chi_{s}, \alpha_{s}^{\prime}=\left(\alpha_{s r}, \alpha_{s z}\right)$, corresponding to $v_{s}^{\prime}, s=1,2$. Then problem (2.12) determines $\tilde{v}_{s}$ corresponding to $\alpha_{s}$, $s=1,2$. Introducing the differences

$$
\begin{gathered}
V^{\prime}=v_{1}^{\prime}-v_{2}^{\prime}, \quad H=h_{1}-h_{2}, \quad Q=q_{1}-q_{2}, \quad W=w_{1}-w_{2}, \quad K=\chi_{1}-\chi_{2}, \\
U=u_{1}-u_{2}, \quad A_{r}=\alpha_{1 r}-\alpha_{2 r}, \quad A_{z}=\alpha_{1 z}-\alpha_{2 z}, \quad \tilde{V}=\tilde{v}_{1}-\tilde{v}_{2},
\end{gathered}
$$

we see that they are solutions to the problems

$$
\begin{gathered}
H_{, t}-\operatorname{div} \mathbb{T}(H, Q)=-V^{\prime} \cdot \nabla h_{1}-v_{2}^{\prime} \cdot \nabla H-H \cdot \nabla v_{1}^{\prime}-h_{2} \cdot \nabla V^{\prime}, \\
\operatorname{div} H=0, \\
\bar{n} \cdot H=0, \quad \bar{n} \cdot \mathbb{D}(H) \cdot \bar{\tau}_{\alpha}=0, \quad \alpha=1,2, \quad \text { on } S^{T}, \\
\left.H\right|_{t=0}=0 ;
\end{gathered}
$$




$$
\begin{aligned}
& W_{, t}-\nu \Delta W+\nu \frac{W}{r^{2}}=-V^{\prime} \cdot \nabla w_{1}-v_{2}^{\prime} \cdot \nabla W \\
& -\frac{V_{r}^{\prime}}{r} w_{1}-\frac{v_{2 r}^{\prime}}{r} W+\frac{1}{r} Q+\frac{2 \nu}{r^{2}} H, \\
& W_{, r}=\frac{1}{R} W \quad \text { on } S_{1}^{T}, \quad W_{, z}=0 \text { on } S_{2}^{T}, \\
& \left.W\right|_{t=0}=0 \text {; } \\
& K_{, t}+V^{\prime} \cdot \nabla \chi_{1}+v_{2}^{\prime} \cdot \nabla K+\left(V_{r, r}^{\prime}+V_{z, z}^{\prime}\right) \chi_{1}+\left(v_{2 r, r}^{\prime}+v_{2 z, z}^{\prime}\right) K \\
& -\nu \Delta K+\nu \frac{K}{r^{2}}=\frac{2 \nu}{r}\left(-H_{\varphi, z}+\frac{1}{r} H_{z, \varphi}\right)-\frac{1}{r}\left(W_{, z} h_{1 r}+w_{2, z} H_{r}\right) \\
& -W_{, r} h_{1 z}-w_{2, r} H_{z}+\frac{W}{r} h_{1 z}+\frac{w_{2}}{r} H_{z}+\frac{2}{r} W u_{1}+\frac{2}{r} w_{2} U, \\
& K=0 \quad \text { on } S^{T},\left.K\right|_{t=0}=0 ; \\
& U_{, t}+V^{\prime} \cdot \nabla u_{1}+v_{2}^{\prime} \cdot \nabla U+\frac{V_{r}^{\prime}}{r} u_{1}+\frac{v_{2 r}^{\prime}}{r} U-\nu \Delta U+\nu \frac{U}{r^{2}} \\
& =-V_{, z}^{\prime} \cdot \nabla w_{1}-v_{2, z}^{\prime} \cdot \nabla W-\frac{V_{r, z}^{\prime}}{r} w_{1}-\frac{v_{2 r, z}^{\prime}}{r} W+\frac{1}{r} Q_{, z}+\frac{2 \nu}{r^{2}} H_{, z}, \\
& U_{, r}=\frac{1}{R} U \quad \text { on } \quad S_{1}^{T}, \quad U=0 \quad \text { on } \quad S_{2}^{T},\left.\quad U\right|_{t=0}=0 ; \\
& A_{r, t}+V^{\prime} \cdot \nabla \alpha_{1 r}+v_{2}^{\prime} \cdot \nabla A_{r}-\left(V_{r, r}^{\prime} \alpha_{1 r}+V_{r, z}^{\prime} \alpha_{1 z}\right) \\
& -\left(v_{2 r, r}^{\prime} A_{r}+v_{2 r, z}^{\prime} A_{z}\right)-\frac{K}{r} h_{1 r}-\frac{\chi_{2}}{r} H_{r}-\nu \Delta A_{r}+\nu \frac{A_{r}}{r^{2}} \\
& =-\frac{2 \nu}{r^{2}}\left(H_{r, z}-H_{z, r}\right), \\
& A_{r, r}=-\frac{1}{R^{2}} H_{z}-\frac{1}{R} W_{, z} \quad \text { on } \quad S_{1}^{T}, \quad A_{r}=0 \quad \text { on } \quad S_{2}^{T},\left.\quad A_{r}\right|_{t=0}=0 \text {; } \\
& A_{z, t}+V^{\prime} \cdot \nabla \alpha_{1 z}+v_{2}^{\prime} \cdot \nabla A_{z}-\left(V_{r, r}^{\prime} \alpha_{1 r}+V_{z, z}^{\prime} \alpha_{1 z}\right)-\left(v_{2 z, r}^{\prime} A_{r}+v_{2 z, z}^{\prime} A_{z}\right) \\
& -\frac{K}{r} h_{1 z}-\frac{\chi_{2}}{r} H_{z}-\nu \Delta A_{z}=0, \\
& A_{z}=\frac{2}{R} W \quad \text { on } \quad S_{1}^{T}, \quad A_{z, z}=0 \quad \text { on } \quad S_{2}^{T},\left.\quad A_{z}\right|_{t=0}=0 \text {; } \\
& \operatorname{rot} \tilde{V}=\bar{A} \text {, } \\
& \operatorname{div} \tilde{V}=0 \\
& \left.\tilde{V} \cdot \bar{n}\right|_{S}=0,
\end{aligned}
$$

where $\bar{A}=\left(A_{r}, K, A_{z}\right), A^{\prime}=\left(A_{r}, A_{z}\right)$.

To obtain estimates for solutions to problems (5.3)-(5.9), we use Lemmas 4.1 and 4.2. Hence,

$$
\left\|v_{s}^{\prime}\right\|_{\mathfrak{M}\left(\Omega^{t}\right)} \leq A, \quad s=1,2 .
$$

In view of (5.10) we obtain for solutions to problems $(2.5)-(2.11)$ the estimates 


$$
\begin{aligned}
& \left\|h_{s}\right\|_{2,-\left(1+\varepsilon_{*}\right), \Omega^{t}}+\left\|q_{s}\right\|_{L_{2}\left(0, t ; H_{-\left(1+\varepsilon_{*}\right)}^{1}(\Omega)\right)} \leq \varphi(A), \\
& \left\|\chi_{s} / r\right\|_{V_{2}^{0}\left(\Omega^{t}\right)} \leq \varphi(A), \\
& \left\|u_{s}\right\|_{V_{2,-\mu}^{0}\left(\Omega^{t}\right)} \leq \varphi(A), \quad \mu \in(0,1), \\
& \left\|w_{s}\right\|_{L_{\infty}\left(0, t: H_{0}^{1}(\Omega)\right)}+\left|w_{s, t}\right|_{2, \Omega^{t}} \leq \varphi(A), \\
& \left\|\alpha_{s}^{\prime}\right\|_{V_{2}^{0}\left(\Omega^{t}\right)} \leq \varphi(A), \quad s=1,2, \quad t \leq T,
\end{aligned}
$$

where $V_{2,-\mu}^{0}\left(\Omega^{T}\right)$ is $V_{2}^{0}\left(\Omega^{T}\right)$ with the $L_{2}(\Omega)$ norm replaced by $L_{2,-\mu}(\Omega)$.

In view of (5.11) we obtain for solutions to problems $(5.3)-(5.8)$ the inequalities

$$
\begin{aligned}
& \|H\|_{2,-\left(1+\varepsilon_{*}\right), \Omega^{t}}+\|Q\|_{L_{2}\left(0, t ; H_{-\left(1+\varepsilon_{*}\right)}^{1}(\Omega)\right)} \leq \varepsilon(A)\left\|V^{\prime}\right\|_{\mathfrak{M}\left(\Omega^{t}\right)}, \\
& \|K / r\|_{V_{2}^{0}\left(\Omega^{t}\right)} \leq \varphi(A, t)\left(\left\|V^{\prime}\right\|_{\mathfrak{M}\left(\Omega^{t}\right)}+\sup _{t}\|W(t)\|_{1,0, \Omega}\right. \\
& \left.+\|U\|_{L_{2}\left(0, t ; L_{3,-1}(\Omega)\right)}\right), \\
& \|U\|_{V_{2,-\mu}^{0}\left(\Omega^{t}\right)} \leq \varphi(A, t)\left[\left\|V^{\prime}\right\|_{\mathfrak{M}\left(\Omega^{t}\right)}+\sup _{t}\|W\|_{1,0, \Omega}\right], \quad \mu \in(0,1), \\
& \sup _{t}\left(\|W(t)\|_{1,0, \Omega}+|W(t)|_{2, \Omega}\right)+\left|W_{, t}\right|_{2, \Omega^{t}} \leq \varphi(A)\left\|V^{\prime}\right\|_{\mathfrak{M}\left(\Omega^{t}\right)}, \\
& \left\|A^{\prime}\right\|_{V_{2}^{0}\left(\Omega^{t}\right)} \leq \varphi(A, t)\left\|V^{\prime}\right\|_{\mathfrak{M}\left(\Omega^{t}\right)} \text {. }
\end{aligned}
$$

From (5.12) we have

$$
\|\bar{A}\|_{V_{2}^{0}\left(\Omega^{t}\right)} \leq \varphi(A, t)\left\|V^{\prime}\right\|_{\mathfrak{M}\left(\Omega^{t}\right)}
$$

so problem (5.9) implies

$$
\|\tilde{V}\|_{\mathfrak{M}_{0}\left(\Omega^{t}\right)} \leq \varphi(A, t)\left\|V^{\prime}\right\|_{\mathfrak{M}\left(\Omega^{t}\right)} .
$$

Let $V=v_{1}-v_{2}, P=p_{1}-p_{2}$. Then problem (5.1) implies

$$
\begin{gathered}
V_{, t}-\operatorname{div} \mathbb{T}(V, P)=-\lambda\left(V \cdot \nabla v_{1}+v_{2} \cdot \nabla V\right), \\
\operatorname{div} V=0 \\
\left.\bar{n} \cdot V\right|_{S}=0,\left.\quad \bar{n} \cdot \mathbb{T}(V, P) \cdot \bar{\tau}_{\alpha}\right|_{S}=0, \quad \alpha=1,2, \\
\left.V\right|_{t=0}=0 .
\end{gathered}
$$

Hence, (5.13) yields

$$
\|V\|_{\mathfrak{M}_{*}\left(\Omega^{t}\right)} \leq \varphi(A)\|\tilde{V}\|_{\mathfrak{M}_{0}\left(\Omega^{t}\right)} \leq \varphi(A, t)\left\|V^{\prime}\right\|_{\mathfrak{M}\left(\Omega^{t}\right)} .
$$

This implies continuity of the mapping $\Phi$ and ends the proof.

6. Appendix. In this section we consider the problem

$$
\begin{array}{cl}
u_{t}-\Delta u=0 & \text { in } \Omega^{T}, \\
\left.u\right|_{S}=\varphi & \text { on } S^{T}, \\
\left.u\right|_{t=0}=0 & \text { in } \Omega .
\end{array}
$$

First we examine problem (6.1) in the half-space $x_{3}>0$.

The fundamental solution to $(6.1)_{1}$ has the form

$$
\begin{aligned}
& \Gamma(x, t)=\frac{1}{(4 \pi t)^{3 / 2}} e^{-\frac{x^{2}}{4 t}}, \quad t>0, \\
& \Gamma(x, t)=0, \quad t<0 .
\end{aligned}
$$


Then a solution to problem (6.1) in the half-space $x_{3}>0$ has the form

$$
u(x, t)=-2 \int_{0}^{t} d t^{\prime} \int_{\mathbb{R}^{2}} \frac{\partial \Gamma\left(x^{\prime}-y^{\prime}, x_{3}, t-t^{\prime}\right)}{\partial x_{3}} \varphi\left(y^{\prime}, t^{\prime}\right) d y^{\prime} .
$$

Using the form of $\Gamma$,

$$
\Gamma\left(x^{\prime}-y^{\prime}, x_{3}, t-t^{\prime}\right)=\frac{1}{\left[4 \pi\left(t-t^{\prime}\right)\right]^{3 / 2}} \exp \left[-\frac{\left(x^{\prime}-y^{\prime}\right)^{2}+x_{3}^{2}}{4\left(t-t^{\prime}\right)}\right]
$$

we obtain $(6.3)$ in the form

$$
u(x, t)=\frac{x_{3}}{(4 \pi)^{3 / 2}} \int_{0}^{t} \frac{d t^{\prime}}{\left(t-t^{\prime}\right)^{5 / 2}} \int_{\mathbb{R}^{2}} \exp \left[-\frac{\left(x^{\prime}-y^{\prime}\right)^{2}+x_{3}^{2}}{4\left(t-t^{\prime}\right)}\right] \varphi\left(y^{\prime}, t^{\prime}\right) d y^{\prime},
$$

where $x=\left(x^{\prime}, x_{3}\right), x^{\prime}=\left(x_{1}, x_{2}\right), y^{\prime}=\left(y_{1}, y_{2}\right)$.

To obtain an estimate we introduce new variables $\tau=t-t^{\prime}, x^{\prime}-y^{\prime}=z^{\prime}$. Hence we get

$$
u(x, t)=\frac{x_{3}}{(4 \pi)^{3 / 2}} \int_{0}^{t} \frac{d \tau}{\tau^{5 / 2}} \int_{\mathbb{R}^{2}} \exp \left[-\frac{z^{\prime 2}+x_{3}^{2}}{4 \tau}\right] \varphi\left(x^{\prime}-z^{\prime}, t-\tau\right) d z^{\prime} .
$$

First we estimate

$$
\begin{aligned}
& \left\|u\left(x_{3}\right)\right\|_{L_{q}\left(0, T, L_{p}\left(\mathbb{R}^{2}\right)\right)} \\
& \quad \leq \frac{x_{3}}{(4 \pi)^{3 / 2}} \int_{0}^{\infty} \frac{d \tau}{\tau^{5 / 2}} \int_{\mathbb{R}^{2}} \exp \left[-\frac{z^{\prime 2}+x_{3}^{2}}{4 \tau}\right]\|\varphi\|_{L_{q}\left(0, T ; L_{p}\left(\mathbb{R}^{2}\right)\right)} d z^{\prime} .
\end{aligned}
$$

Next we calculate

$$
\begin{aligned}
\left(\int_{0}^{a}\left\|u\left(x_{3}\right)\right\|_{L_{q}\left(0, T ; L_{p}\left(\mathbb{R}^{2}\right)\right)}^{p} d x_{3}\right)^{1 / p} \leq & c\left(\int_{0}^{a}\left|x_{3} \int_{0}^{\infty} e^{-\frac{x_{3}^{2}}{4 \tau}} \frac{d \tau}{\tau^{5 / 2}} \int_{\mathbb{R}^{2}} e^{-\frac{z^{\prime 2}}{4 \tau}} d z^{\prime}\right|^{p} d x_{3}\right)^{1 / p} \\
& \times\|\varphi\|_{L_{q}\left(0, T ; L_{p}\left(\mathbb{R}^{2}\right)\right)} .
\end{aligned}
$$

Let us calculate the integral on the r.h.s. of (6.8). Introducing the new variables

$$
z^{\prime \prime}=\frac{z^{\prime}}{2 \sqrt{\tau}}, \quad d z^{\prime}=(2 \sqrt{\tau})^{2} d z^{\prime \prime},
$$

the integral takes the form

$$
\begin{gathered}
\left(\int_{0}^{a}\left|x_{3} \int_{0}^{\infty} e^{-\frac{x_{3}^{2}}{4 \tau}} \frac{d \tau}{\tau^{5 / 2}} 4 \tau \int_{\mathbb{R}^{2}} e^{-z^{\prime \prime 2}} d z^{\prime \prime}\right|^{p} d x_{3}\right)^{1 / p} \\
\leq c\left(\int_{0}^{a}\left|x_{3} \int_{0}^{\infty} e^{-\frac{x_{3}^{2}}{4 \tau}} \frac{d \tau}{\tau^{3 / 2}}\right|^{p} d x_{3}\right)^{1 / p} \equiv I_{1} .
\end{gathered}
$$

Introducing $z=\frac{x_{3}}{2 \sqrt{\tau}}, d z=-\frac{x_{3}}{4 \tau^{3 / 2}} d \tau$ yields

$$
I_{1} \leq c\left(\int_{0}^{a} d x_{3}\left|\int_{0}^{\infty} e^{-z^{2}} d z\right|^{p}\right)^{1 / p} \leq c\left(\int_{0}^{a} d x_{3}\right)^{1 / p} \leq c a^{1 / p} .
$$

Finally, for $a<\infty$ the estimate

$$
\|u\|_{L_{p}\left(0, a ; L_{q}\left(0, T ; L_{p}\left(\mathbb{R}^{2}\right)\right)\right)} \leq c\|\varphi\|_{L_{q}\left(0, T ; L_{p}\left(\mathbb{R}^{2}\right)\right)}
$$

holds. 
Let us consider the case of bounded cylindrical domain with the boundary $S=S_{1} \cup S_{2}$. Then solutions to (6.1) are expressed in the form (for more details see [4, Ch. 4, Sect. 16])

$$
u(x, t)=\int_{0}^{t} d \tau \int_{S} n_{i}(\xi) \frac{\partial \Gamma(x-\xi, t-\tau)}{\partial \xi_{i}} \mu(\xi, \tau) d S_{\xi},
$$

where $\mu(\xi, \tau)$ is a density and $\bar{n}=\left(n_{1}, n_{2}, n_{3}\right)$ the unit normal vector to $S$.

Considering the limit

$$
\lim _{x \rightarrow \eta \in S} u(x, t)=\int_{0}^{t} d \tau \int_{S} n_{i}(\xi) \frac{\partial \Gamma(\eta-\xi, t-\tau)}{\partial \xi_{i}} \mu(\xi, \tau) d S_{\xi} \mp \frac{1}{2} \mu(\eta, t)
$$

we obtain the integral equation for $\mu$

$$
\mu(\eta, t)=2 \int_{0}^{t} d \tau \int_{S} n_{i}\left(\xi_{i}\right) \frac{\partial \Gamma(\eta-\xi, t-\tau)}{\partial \xi_{i}} \mu(\xi, \tau) d S_{\xi}-2 \varphi(\eta, t)
$$

in the case of the interior problem. This is a Volterra type equation with a weakly singular kernel. Hence $\mu$ has the same regularity as $\varphi$. Applying a partition of unity to the integral over $S$ in (6.10), using estimate (6.9) and the Minkowski inequality (see [2, Ch. 1, Sect. 2])

$$
\|u\|_{L_{q}\left(0, T ; L_{p}\left(\mathbb{R}^{2} \times(0, a)\right)\right.} \leq c\|u\|_{L_{p}\left(0, a ; L_{q}\left(0, T ; L_{p}\left(\mathbb{R}^{2}\right)\right)\right.}
$$

which holds for $1 \leq p \leq q \leq \infty$, we get

Lemma 6.1. Let $\varphi \in L_{q}\left(0, T ; L_{p}(S)\right), 1 \leq p \leq q \leq \infty$. Then solutions of (6.1) are such that $u \in L_{q}\left(0, T ; L_{p}(\Omega)\right)$ and

$$
\|u\|_{L_{q}\left(0, T ; L_{p}(\Omega)\right)} \leq c\|\varphi\|_{L_{q}\left(0, T ; L_{p}(S)\right)} .
$$

Problem (6.1) in the half-space $x_{3}>0$ has the form

$$
\begin{aligned}
u_{t}-\Delta u=0, & x_{3}>0, \\
u=\varphi, & x_{3}=0, \\
\left.u\right|_{t=0}=0, & x_{3}>0 .
\end{aligned}
$$

To solve (6.13) we apply the Fourier-Laplace transform

$$
\tilde{u}\left(\xi, x_{3}, s\right)=\int_{0}^{\infty} d t e^{-s t} \int_{\mathbb{R}^{2}} e^{-i x^{\prime} \cdot \xi} u(x, t) d x^{\prime},
$$

where $s=\gamma+i \xi_{0}$, Re $s=\gamma>0, x^{\prime}=\left(x_{1}, x_{2}\right), \xi=\left(\xi_{1}, \xi_{2}\right), x^{\prime} \cdot \xi=x_{1} \xi_{1}+x_{2} \xi_{2}$. After transformation (6.14) problem (6.13) takes the form

$$
\begin{gathered}
\left(-\partial_{x_{3}}^{2}+\tau^{2}\right) \tilde{u}=0, \\
\left.\tilde{u}\right|_{x_{3}=0}=\tilde{\varphi},
\end{gathered}
$$

where $\tau^{2}=s+|\xi|^{2}, \arg \tau \in\left(-\frac{\pi}{4}, \frac{\pi}{4}\right)$.

To obtain (6.15) we used problem (6.13) with solution $u$ extended by zero for $t<0$. Looking for solutions of (6.15) vanishing for $x_{3}=\infty$ we obtain

$$
\tilde{u}=\tilde{\varphi} e^{-\tau x_{3}} \text {. }
$$

Let us consider the norm (see $[3,6])$

$$
\|u\|_{1, \gamma, \mathbb{R}_{+}^{3} \times \mathbb{R}}^{2}=\int_{\mathbb{R}^{2}} d \xi \int_{-\infty}^{\infty} d \xi_{0} \int_{\mathbb{R}_{+}} d x_{3}\left[\left|\tilde{u}\left(\xi, x_{3}, s\right)\right|^{2}\left(|\xi|^{2}+|s|\right)+\left|\partial_{x_{3}} \tilde{u}\left(\xi, x_{3}, s\right)\right|^{2}\right] \equiv I_{1} .
$$


Using the estimates (see $[3,6])$

$$
\int_{0}^{\infty}\left|e^{-\tau x_{3}}\right|^{2} d x_{3} \leq c|\tau|^{-1}, \quad \int_{0}^{\infty}\left|\partial_{x_{3}} e^{-\tau x_{3}}\right|^{2} d x_{3} \leq c|\tau|,
$$

we obtain for solutions (6.16) the estimate

$$
\|u\|_{1, \gamma, \mathbb{R}_{+}^{3} \times \mathbb{R}}^{2} \leq c \int_{\mathbb{R}^{2}} d \xi \int_{\mathbb{R}} d \xi_{0}|\tilde{\varphi}|^{2}|\tau|=c\|\varphi\|_{1 / 2, \gamma, \mathbb{R}^{2} \times \mathbb{R}}^{2} .
$$

From $[3,6]$ we know the equivalence

$$
c_{1}\|u\|_{H^{l, 1 / 2}} \leq\|u\|_{l, \gamma} \leq c_{2}\|u\|_{H^{l, 1 / 2}} .
$$

Hence (6.17) assumes the form

$$
\|u\|_{W_{2}^{1,1 / 2}\left(\mathbb{R}_{+}^{3} \times \mathbb{R}\right)} \leq c\|\varphi\|_{W_{2}^{1 / 2,1 / 4}\left(\mathbb{R}^{2} \times \mathbb{R}\right)} .
$$

To obtain an estimate of the form (6.18) for solutions of (6.1) we examine (6.10). Let $\left\{\psi_{\alpha}(x)\right\}$ be a partition of unity for $\Omega$ and $\left\{\varphi_{\beta}(s)\right\}, s \in S$, be a partition of unity for $S$. Then, from (6.10) we have

$$
\begin{aligned}
\psi_{\alpha} u & =\psi_{\alpha} \int_{0}^{t} d t \int_{S} \sum_{\beta} \varphi_{\beta}(\xi) n_{i}(\xi) \frac{\partial \Gamma(x-\xi, t-\tau)}{\partial \xi_{i}} \mu(\xi, \tau) d S_{\xi} \\
& =\psi_{\alpha} \sum_{\beta} \int_{0}^{t} d t \int_{S \cap \operatorname{supp}_{\beta}} \varphi_{\beta}(\xi) n_{i}(\xi) \frac{\partial \Gamma(x-\xi, t-\tau)}{\partial \xi_{i}} \mu(\xi, \tau) d S_{\xi} .
\end{aligned}
$$

We introduce the local coordinates $\eta=\left(\eta_{1}, \eta_{2}, \eta_{3}\right)$ on $S \cap \operatorname{supp} \varphi_{\beta}$, and transform $S \cap$ $\operatorname{supp} \varphi_{\beta}$ on the plane $\eta_{3}=0$. Moreover, in $\operatorname{supp} \psi_{\alpha}$ we introduce a local coordinate system $y=\left(y_{1}, y_{2}, y_{3}\right)$ such that $y_{3}=0$ is the plane $\eta_{3}=0$. Then (6.19) takes the form

$$
u^{\prime}\left(y_{1}, y_{2}, y_{3}\right)=\int_{0}^{t} d \tau \int_{\mathbb{R}^{2}} \frac{\partial \Gamma\left(y^{\prime}-\eta^{\prime}, y_{3}, t-\tau\right)}{\partial y_{3}} \mu^{\prime}\left(\eta^{\prime}, \tau\right) d \eta^{\prime},
$$

where $\mu^{\prime}$ and $u^{\prime}$ have compact supports and $y^{\prime}=\left(y_{1}, y_{2}\right), \eta^{\prime}=\left(\eta_{1}, \eta_{2}\right)$. Moreover, we have

$$
\left\|\mu^{\prime}\right\|_{W_{2}^{1 / 2,1 / 4}\left(\mathbb{R}^{2} \times \mathbb{R}_{+}\right)} \leq c\|\mu\|_{W_{2}^{1 / 2,1 / 4}\left(S^{T}\right)} .
$$

Formula (6.20) describes a solution of the problem

$$
\begin{gathered}
u_{, t}^{\prime}-\Delta u^{\prime}=0, \\
\left.u^{\prime}\right|_{x_{3}}=\mu^{\prime},\left.\quad u^{\prime}\right|_{t=0}=0 .
\end{gathered}
$$

In view of (6.18) we have for the solutions of (6.22) the estimate

$$
\left\|u^{\prime}\right\|_{L_{2}\left(\mathbb{R}_{+} ; H^{1}\left(\mathbb{R}^{2}\right)\right)} \leq c\left\|\mu^{\prime}\right\|_{W_{2}^{1 / 2,1 / 4}\left(\mathbb{R}^{2} \times \mathbb{R}_{+}\right)} .
$$

Then for the solutions of the integral equation (6.11) we have

$$
\|\mu\|_{W_{2}^{1 / 2,1 / 4}\left(S^{T}\right)} \leq c\|\varphi\|_{W_{2}^{1 / 2,1 / 4}\left(S^{T}\right)}
$$

Using (6.23), (6.21) and (6.24) and summing over all subdomains of the introduced partitions of unity we obtain the following estimate for solutions to problem (6.1),

$$
\|u\|_{L_{2}\left(0, T ; H^{1}(\Omega)\right)} \leq c\|\varphi\|_{W_{2}^{1 / 2,1 / 4}\left(S^{T}\right)} .
$$

Hence, we have 
Lemma 6.2. Let $\varphi \in W_{2}^{1 / 2,1 / 4}\left(S^{T}\right)$. Then there exists a solution to problem (6.1) such that $u \in L_{2}\left(0, T ; H^{1}(\Omega)\right)$ and (6.25) holds.

\section{References}

[1] W. Alame, On the existence of solutions for the nonstationary Stokes system with slip boundary conditions, Appl. Math. 32 (2005), 195-223.

[2] O. V. Besov, V. P. Il'in and S. M. Nikol'skiı̌, Integral Representations of Functions and Imbedding Theorems, Nauka, Moscow, 1975 (in Russian).

[3] M. Burnat and W. M. Zajączkowski, On local motion of a compressible barotropic viscous fluid with the boundary slip condition, Topol. Meth. Nonlinear Anal. 10 (1997), 195-223.

[4] O. A. Ladyzhenskaya, V. A. Solonnikov and N. N. Ural'tseva, Linear and Quasilinear Equations of Parabolic Type, Nauka, Moscow, 1967 (in Russian).

[5] V. G. Maz'ya and B. A. Plamenevskiū, $L_{p}$-estimations for solutions of elliptic boundary value problems in domains with edges, Trudy Mosk. Mat. Obshch. 37 (1978), 49-93 (in Russian).

[6] V. A. Solonnikov, An initial boundary value problem for the Stokes system that arises in the study of a problem with a free boundary, Trudy Mat. Inst. Steklov. 188 (1990), 150-180 (in Russian); English translation: Proc. Steklov Inst. Math. 3 (1991), 191-239.

[7] W. M. Zajączkowski, Global special regular solutions to the Navier-Stokes equations in a cylindrical domain under boundary slip conditions, Gakuto Series in Math. 21 (2004), $1-188$.

[8] W. M. Zajączkowski, Existence of solutions to the Stokes system in $H_{-\mu-1}^{2,1}\left(\Omega^{T}\right), \mu \in(0,1)$, preprint. 
